\title{
Structure design and mechanism analysis of silicon anode for lithium-ion batteries
}

\author{
Xiang Chen, Haixia Li, Zhenhua Yan*, Fangyi Cheng and Jun Chen
}

\begin{abstract}
Silicon-based material is one of the most promising substitutes of widely used graphite anodes for the next generation Li-ion batteries due to its high theoretical capacity, low working potential, environmental friendliness, and abundant natural resource. However, the huge volume expansion and serious interfacial side reactions during lithiation and delithiation progresses of the silicon anode are the key issues which impede their further practical applications. Rational designs of silicon nanostructures are effective ways to address these problems. In this progress report, we firstly highlight the fundamental scientific problems, and then focus on recent progresses in design, preparation, in-situ characterization methods and failure mechanism of nanostructured silicon anode for high capacity lithium battery. We also summarize the key lessons from the successes so far and offer perspectives and future challenges to promote the applications of silicon anode in practical lithium batteries.
\end{abstract}

Keywords: silicon anode, Li-ion batteries, structure design, in situ characterization, mechanism analysis

\section{INTRODUCTION}

Rechargeable lithium-ion batteries (LIBs), as one of the energy storage technologies, are the key part of the contemporary energy system which is closely coupled with renewable energy generation, transmission and utilization [1-4]. Meanwhile, due to the high energy density, long lifespan and environmental friendliness, LIBs have been highly developed over the past decades and widely used in our daily life including portable electronics, electrical transportation, and even grid energy storage [5-7]. However, the current most mature LIBs cannot ever meet the ever-increasing demands [8-9]. Thus, a lot of new electrode materials with different storage mechanisms have been explored to improve the battery performance and reduce the cost [10-13]. Among them, alloy-type Si anode is one of the outstanding examples demonstrating great promise and broad research interest because of its high capacity $\left(4200 \mathrm{~mA} \mathrm{~h} \mathrm{~g}^{-1}\right)$, low operation potential $\left(0.2-0.3 \mathrm{~V} v s . \mathrm{Li}^{+} / \mathrm{Li}\right)$, environmental friendliness, and high abundance [14]. Therefore, Si appears to be a very promising anode material for the next generation battery, especially in electric vehicles which require to be powered by LIBs with higher energy density and less safety issues [15-17].

However, the alloy reaction mechanisms of silicon are significantly different from those of conventional layered electrodes. There are many challenges that emerge during lithiation/delithiation progress [18-20] (Fig. 1): complete destruction of crystal structure producing huge volume expansion (ca. 420\%) at the electrode and cell level, chemical bond breaking/reformation with serious interfacial side reactions, and relatively low intrinsic electronic conductivity (ca. $10^{-3} \mathrm{~S} \mathrm{~cm}^{-1}$ ) of silicon, which will limit the cycling stability, Coulombic efficiency (CE) and rate capability, and thus severely impede their further practical applications. In the past several years, significant breakthroughs have been achieved by combining advanced nanomaterial preparation method with in-situ characterization technologies to improve the electrochemical performance and gain a better understanding of the mechanism under the real working condition of $\mathrm{Si}$ anode [21]. Although there have been some excellent reviews in this topic [22-23], the rapid development and newly emerging research results are achieved in the last six to seven years, and more than 700 papers are published every year (Fig. 2). It is essential to summarize the developing trend and direction of silicon anode in recent

Key Laboratory of Advanced Energy Materials Chemistry (Ministry of Education), Renewable Energy Conversion and Storage Center, College of Chemistry, Nankai University, Tianjin 300071, China

*Corresponding author (email: yzh@nankai.edu.cn) 
years to provide some revelation for the further applications.

In this review, we first briefly discuss the fundamental scientific challenges and solutions as well as the development history of silicon anodes, and then focus on the recent progress of rational structure designing; specific examples are provided to illustrate these fundamental design principles. We also highlight in-situ techniques utilized for in-depth understanding the silicon anode performance and fading mechanisms, including atomic force microscopy (AFM), transmission electron microscopy (TEM), nuclear magnetic resonance (NMR), and $\mathrm{X}$-Ray powder diffraction (XRD). Finally, the major challenges and opportunities on silicon based anode materials are presented.

\section{BASIC INTRODUCTION OF SILICON ANODE}

\section{Fundamental scientific problems}

Silicon (Si) commonly exists in silicates and silica, which are widely found in rocks, gravel, and dust, but it rarely appears in the form of elemental silicon in nature. Silicon atom locates in IV main group of the periodic table with an atomic number of 14 . The four valence electrons in the outermost layer make silicon atoms in a metastable structure, which plays a dominant role in conductivity and other properties. The crystalline silicon is three-dimensional (3D) diamond structure in cubic $F d-3 m$ space group with lattice constants of $5.431 \AA$, as shown in Fig. 3a. Silicon is bonded to four equivalent $\mathrm{Si}$ atoms to form corner-sharing $\mathrm{SiSi}_{4}$ tetrahedra. Chemical properties of silicon are relatively stable. It is difficult to react with other substances (except the hydrogen fluoride and lye) at room temperature. Commercial silicon is usually divided into metallic silicon and semiconductor silicon. Metallic silicon is mainly used to make compounds of poly-

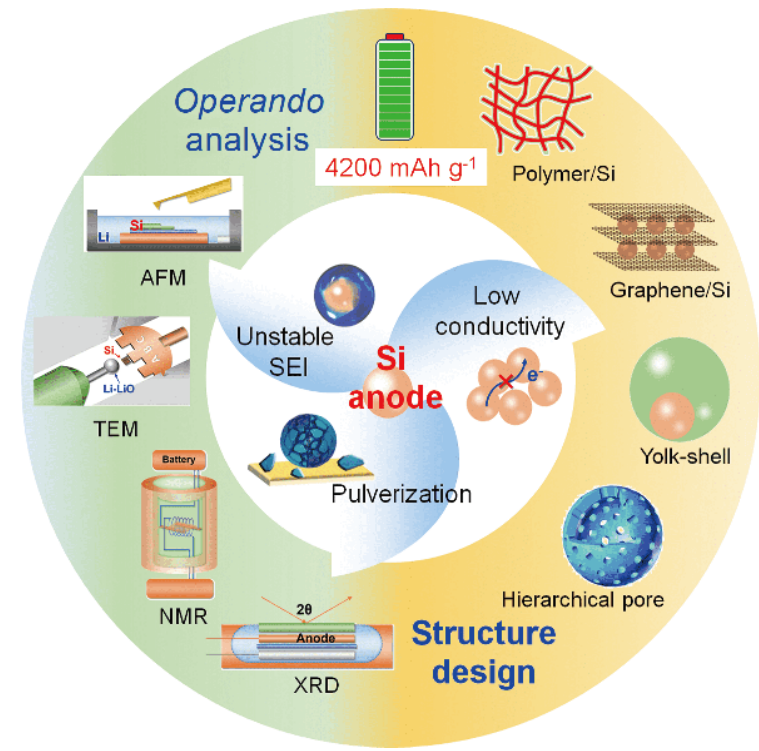

Figure 1 Overview of silicon-based anode for LIBs: fundamental challenges, recent structure design strategies toward commercialization, and failure mechanism analysis technologies.

crystalline silicon, monocrystalline silicon, silicon-aluminum alloy and silicon-steel alloy. Semiconductor silicon is used for producing semiconductor devices. Silicon-based materials have been widely used in aerospace, electronics, electrical, construction, transportation, energy, chemical, textile, food, light industry, medical, agriculture and other industries [24].

When $\mathrm{Si}$ is applied as anode materials for LIBs, it is important to know the properties of Li. Lithium is the lightest metal element with an atomic number of 3, two electrons in the $\mathrm{K}$ layer and one in the L layer. Since lithium has a high charge density and a stable helium double shell, it is easy to polarize other molecules or ions, but not easy to polarize itself. Lithium metal has a typical body centered cubic structure as shown in Fig. 3b. When

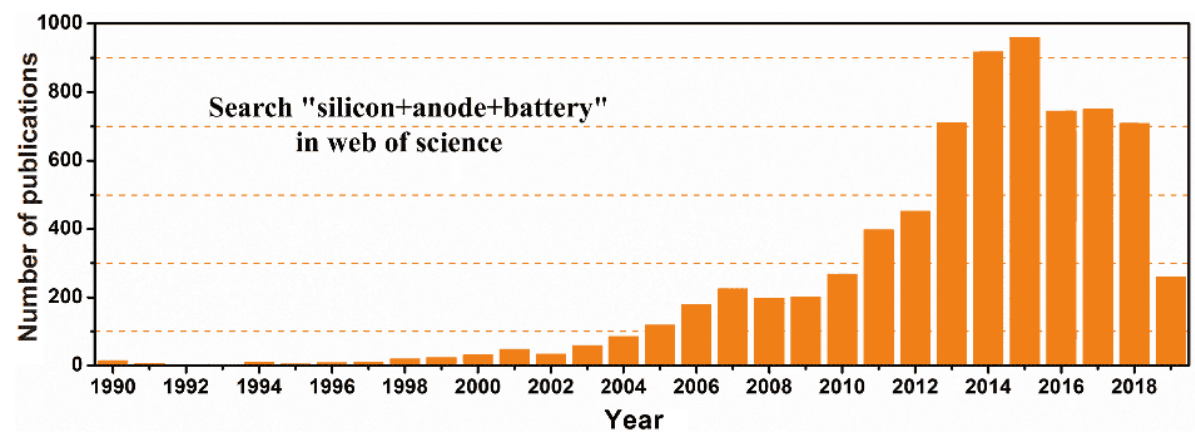

Figure 2 Statistical analysis of the publications on silicon anode in last three decades. The data was up to May 1, 2019. 
a
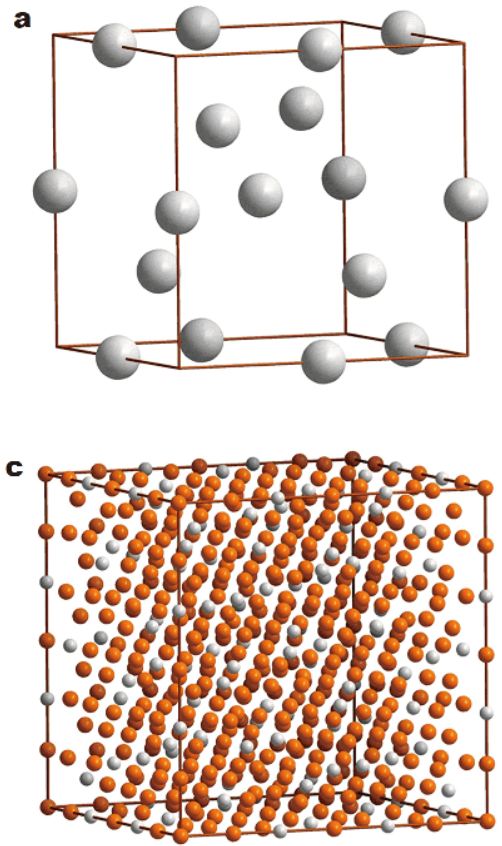

b

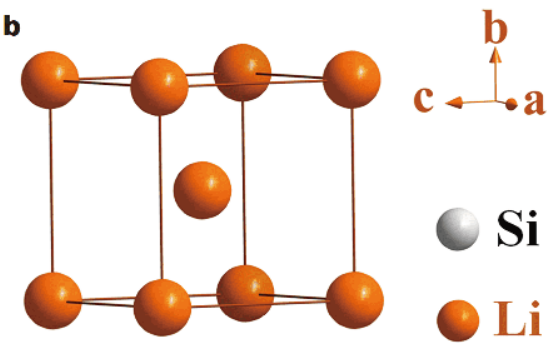

d

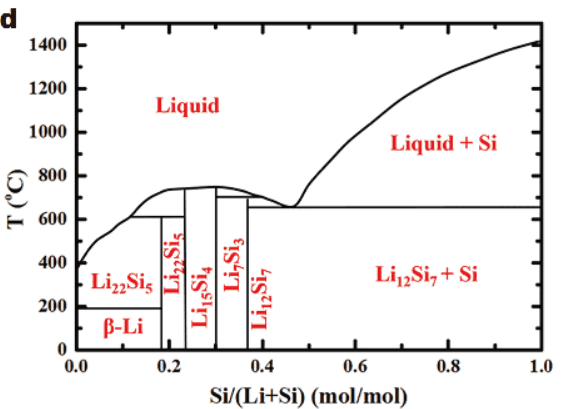

e

$$
\begin{aligned}
4 \mathrm{Si}+15 \mathrm{Li}^{+}+15 \mathrm{e}^{-} & \mathrm{Li}_{15} \mathrm{Si}_{4} 3580 \mathrm{mAh} \mathrm{g} \\
5 \mathrm{Si}+22 \mathrm{Li}^{+}+22 \mathrm{e}^{-} & \leftrightarrow \mathrm{Li}_{22} \mathrm{Si}_{5} 4200 \mathrm{mAh} \mathrm{g}
\end{aligned}
$$

Figure 3 The crystal structures of silicon (a), lithium (b) and $\mathrm{Li}_{22} \mathrm{Si}_{5}$ (c). Redraw phase diagram of Li-Si system (d). Reprinted with permission from Ref. [24], Copyright 2015, Elsevier. Electrode reactions and theoretical capacity of different alloy products (e).

the temperature drops to $-201^{\circ} \mathrm{C}$, it starts turning into a face-centered cubic structure-the lower the temperature is, the greater the change will be, but not completely change. Lithium has the most negative electrode potential, making it the most active metal. Silicon is an alloy type anode, and each silicon atom can host $4.4 \mathrm{Li}$ atoms at most to form $\mathrm{Li}_{22} \mathrm{Si}_{5}$ (Fig. 3c, e), reaching the maximum theoretical capacity. This mechanism of lithiation is associated with massive volume changes due to the fact that the unit cell volume of cubic $S i$ is $40.88 \AA^{3}$, and $1617 \AA^{3}$ for cubic $\mathrm{Li}_{22} \mathrm{Si}_{5}$ [25]. According to the $\mathrm{Si}$ - $\mathrm{Li}$ system phase diagram as shown in Fig. 3d, a series of $\mathrm{Si}-\mathrm{Li}$ phases exist during the alloying process, such as $\mathrm{LiSi}, \mathrm{Li}_{12} \mathrm{Si}_{7}, \mathrm{Li}_{15} \mathrm{Si}_{4}$, and $\mathrm{Li}_{22} \mathrm{Si}_{5}$.

These crystalline phases possess lower formation energy than the corresponding amorphous phases, which tend to be more kinetically stable. However, the amorphous phases $\mathrm{Li}_{x} \mathrm{Si}$ (with $x=0-3.75$ ) are favorably formed during the electrochemical lithiation. It has been found that when $x$ reaches 3.75 , the amorphous phase will transform to crystalline phase. These amorphous phases and con- tinuous unstable solid electrolyte interphase (SEI) production result in large volume change. The large volume expansion/contraction of Si usually causes cracking and pulverization of $\mathrm{Si}$, resulting in unstable SEI and loss of electrical contact of the current collector [26], which finally lead to low CE and rapid capacity fading. In addition, the $\mathrm{Li}^{+}$insertion-induced large deformation and elastic strain lead to fracture or change in morphology of electrodes, which will significantly slow down the kinetics of lithiation process. When silicon cannot withstand the resulting stress, the cracks will occur due to the brittleness and the material will peel off from the electrode, lose electrical contact, convert to dead weight and thereby decrease the specific capacity of the electrode [27].

\section{History of silicon anode research}

With the passage of time and development of technology, substantial efforts have been made in recent decades to solve the problems of the silicon anode. A timeline of selected important breakthroughs in the silicon anode materials is shown in Fig. 4. Due to the fast capacity 


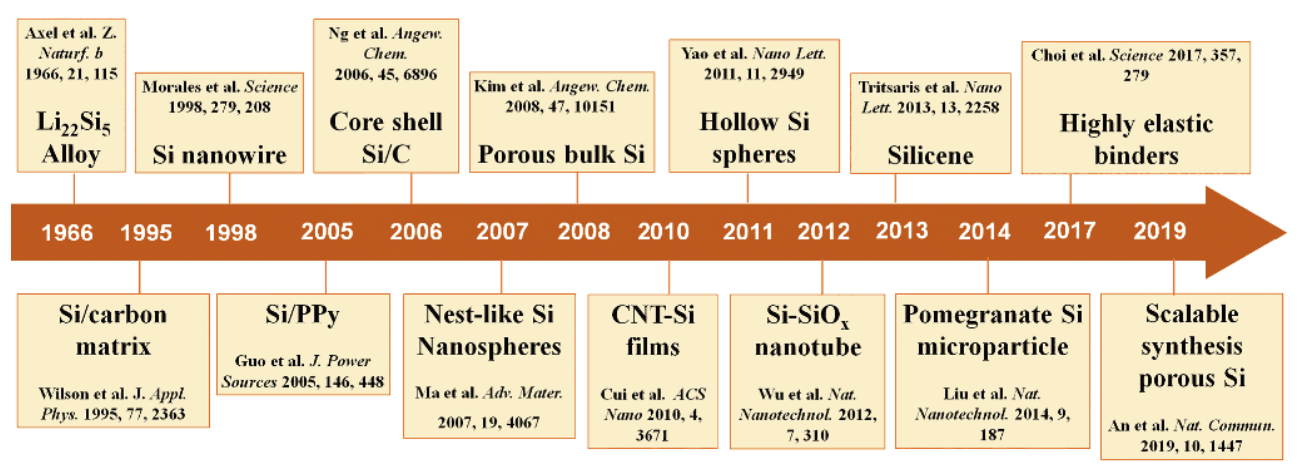

Figure 4 Timeline of selected important breakthroughs in the silicon-based anode.

degradation for limited cycles, silicon anode has been overlooked for a long time since the first discovery of $\mathrm{Li}_{22} \mathrm{Si}_{5}$ alloy in 1966 [28]. In 1971, Dey [29] found that lithium could electrochemically alloy with $\mathrm{Sn}, \mathrm{Pb}, \mathrm{Al}, \mathrm{Au}$, $\mathrm{Pt}, \mathrm{Zn}, \mathrm{Cd}, \mathrm{Ag}$ and $\mathrm{Mg}$ at room temperature. Then Seefurth and Sharma [30] reported the formation of $\mathrm{Li}-\mathrm{Si}$ alloys at high temperature cells. But the reversibility of normal Si powder was very poor due to the volume expansion. Thus, developing effective strategies to resolve the problem of volume expansion has been identified as a central issue of the research on Si-based LIB anodes. In 1995, with the development of nanotechnology, nanosized Si composite had been regarded as the promising method to improve the electrochemical performance. Wilson et al. [31] dispersed nano-sized Si into carbon matrix and obtained a capacity of $600 \mathrm{~mA} \mathrm{~h} \mathrm{~g}^{-1}$, which was about twice that of the commercial graphite anode. $\mathrm{Li}$ et al. [32] prepared $\mathrm{Si} / \mathrm{C}$ composite by manually grinding the mixture of nano-sized $\mathrm{Si}$ and carbon black under ambient condition. The composite exhibited a capacity of $1700 \mathrm{~mA} \mathrm{~h} \mathrm{~g}^{-1}$ even after ten cycles. It was found that nano-sized Si particles played an important role in the improvement of the cyclic stability due to their good plasticity and deformability. In 1998, Lieber's group [33] successfully synthesized $\mathrm{Si}$ nanowires which were considered to be superior to nanoparticles. Benefiting from the continuous electronic pathway, nanowires can efficiently facilitate charge transport. The Si nanowires could realize the theoretical charge capacity of silicon, but the capacity also degraded rapidly after a few cycles. With the development of conductive polymers in the 21st Century, conductive polymers such as polypyrrole (PPy) [34] were used to enhance the charge carrier transportation and inhibit the huge volumetric change. In order to increase the surface area, Si nanotubes were synthesized [35]. The first discharge and charge capacities of the Si nanotubes were 3648 and $3247 \mathrm{~mA} \mathrm{~h} \mathrm{~g}^{-1}$ at the current density of
$600 \mathrm{~mA} \mathrm{~g}^{-1}$ (0.2 C). However, the Si nanotubes had very low mass loading density, which limited the practical applications. Therefore, constructing 3D Si network incorporated with pores became an attractive strategy to resolve the problem of the Si nanotubes. Kim et al. [36] prepared 3D porous bulk Si particles. The $3 \mathrm{D}$ porous structure effectively accommodated stress without pulverization after 100 cycles, and maintained charge capacity of more than $2800 \mathrm{~mA} \mathrm{~h} \mathrm{~g}^{-1}$ at $400 \mathrm{~mA} \mathrm{~g}^{-1}$. Our group also prepared nest-like Si particles composed of hollow Si spheres through solvothermal reaction in 2007, which displayed an initial specific capacity of $3052 \mathrm{~mA} \mathrm{~h} \mathrm{~g}^{-1}$ at the current density of $2000 \mathrm{~mA} \mathrm{~g}^{-1}$, and retained $1095 \mathrm{~mA} \mathrm{~h} \mathrm{~g}^{-1}$ after cycling up to 48 cycles [37]. Because the commonly used heavy metal copper current collector for traditional Si film anode was much heavier than the Si active material itself, it unavoidably increased the total mass of the LIBs. To resolve this problem, a novel anode structure free of heavy metal current collector was designed by integrating a flexible, conductive carbon nanotube (CNT) network into a Si anode [38]. The CNT-Si films showed a high specific charge storage capacity and a good cycling life. Besides the conventional porous structures, nanostructured hollow $\mathrm{Si}$ was also designed and synthesized. Cui's group [39] fabricated hollow $\mathrm{Si}$ nanospheres which exhibited an initial discharge capacity of $2725 \mathrm{~mA} \mathrm{~g}^{-1}$ with less than $8 \%$ capacity degradation every hundred cycles for total 700 cycles at $220 \mathrm{~mA} \mathrm{~g}^{-1}$. The well-designed nanoparticles with a free volume in the hollow particle interior and the porosity in the shell effectively cushioned the volume change and consequently improved the electrochemical performance.

In order to address the SEI stability issue, Cui's group [40] designed a novel double-walled $\mathrm{Si}_{-} \mathrm{SiO}_{x}$ nanotube anode, in which the inner wall was active $\mathrm{Si}$ and the outer wall was the confined $\mathrm{SiO}_{x}$, which still allowed $\mathrm{Li}$ ions to pass through. The outer surface of the Si nanotube was 
prevented from expansion by the oxide shell. The expanding inner surface was not exposed to the electrolyte. This specific structure design helped to stabilize the SEI. The Si nanotube anode exhibited a high specific charge capacity (1780 $\mathrm{mA} \mathrm{h} \mathrm{g}^{-1}$ at $0.2 \mathrm{C}$ ). In 2013 , Wang's group [41] employed the first-principles calculations to investigate the interaction of $\mathrm{Li}$ with $\mathrm{Si}$ in model electrodes of free-standing single-layer and double-layer silicone. Inspired by the structure of pomegranate, Cui's group [42] proposed a hierarchical structured silicon anode where single silicon nanoparticles were encapsulated by a conductive carbon layer that left enough room for expansion and contraction following lithiation and delithiation. As a result of this hierarchical arrangement, the SEI remained stable and spatially confined, resulting in superior cyclability (97\% capacity retention after 1000 cycles). Up to now, scalable synthesis of silicon anodes with good cyclability and low electrode swelling remains a significant challenge.

Tremendous strategies have been proposed to deal with the issues listed above and improve the LIBs performance of silicon-based anode, such as downsizing silicon with various dimensions and microstructures [43-48], compositing with carbon [49-55], doping with metal [56-59], metal oxides [60-63] and conductive polymer modification [64-66]. In the following section, recent progress in these strategies will be discussed in detail.

\section{RATIONAL DESIGNED SILICON ANODES}

\section{Nanostructured Si anode}

Engineering of nanostructure has been proven to be one of the effective ways to buffer the significant volume change of silicon in the charge/discharge process due to their native features: large specific surface area promoting the infiltration between active materials and electrolyte, smaller particle size shortening the diffusion distance of electrons and lithium ions, as well as reducing the inhomogeneous lithium diffusion-induced stress and strain $[14,67]$. Thus, different nanostructures have been designed to improve the electrochemical performance of silicon anodes. In this part, various kinds of silicon nanostructures reported lately are briefly discussed, covering 0 dimensional (0D) nanoparticles, $1 \mathrm{D}$ nanowires, 2D nanosheets and silicene, 3D hierachical nanostructure, hollow and porous structures. Turning the bulk silicon to nanoscale demonstrates one of the efficient strategies to overcome the large expansion/contraction problem by relieving the internal strain. In this regard, Kim et al. [68] reported well-dispersed $0 \mathrm{D}$ Si nanoparticles with various particle sizes by a reverse micelle method for LIB anode. The capacity can still be maintained at $2700 \mathrm{~mA} \mathrm{~h} \mathrm{~g}^{-1}$ after 40 cycles at $0.2 \mathrm{C}$. The highest charge capacity was obtained with a critical size of $10 \mathrm{~nm}$. Another example of such a particle-size-dependent conclusion was confirmed by Liu et al. [69]. By using in-situ TEM technology, the morphological changes of silicon particles with different sizes after the first lithium insertion were compared. It was found that with the decrease of size, silicon exhibited stronger resistance to mechanical strain and its cracking degree decreased. Researchers proposed that $150 \mathrm{~nm}$ was the critical point. Recently, consistent with these conclusions, Lin et al. [70] prepared crystalline Si nanoparticles through a reduction of micro-sized silicon zeolite by metallic $\mathrm{Al}$ in molten $\mathrm{AlCl}_{3}$ at a low temperature of $200^{\circ} \mathrm{C}$, as shown in Fig. 5a. The prepared Si used as an anode for LIBs reached reversible capacities of $2663 \mathrm{~mA} \mathrm{~h} \mathrm{~g}^{-1}$ at $0.5 \mathrm{~A} \mathrm{~g}^{-1}$ after 50 cycles and $870 \mathrm{~mA} \mathrm{~h} \mathrm{~g}^{-1}$ at $3 \mathrm{~A} \mathrm{~g}^{-1}$ after 1000 cycles.

In addition to $0 \mathrm{D}$ silicon nanoparticles, $1 \mathrm{D}$ silicon nanowires can also effectively improve the electrochemical performance. In 2007, Cui's group [71] firstly used Si nanowires vertically aligned on stainless steel substrate with a diameter of $90 \mathrm{~nm}$ as anodes of LIBs by the chemical vapor deposition (CVD) method. In their study, the improved LIB performances were ascribed to the advanced architecture of the Si nanowires electrodes, which provided sufficient space between the nanowires to release the strain from large volume change, and enhanced the electrical contact between $\mathrm{Si}$ nanowires and the substrate. Very recently, Wang et al. [72] used a magnetron sputtering deposition with metal-catalyzed electroless etching technology to produce silicon nanowire film (Fig. 5b), which allowed for achieving the high capacity and excellent cyclability. The prepared anode delivered an initial reversible discharge capacity of $3158 \mathrm{~mA} \mathrm{~h} \mathrm{~g}^{-1}$ and maintained a capacity of $2840 \mathrm{~mA} \mathrm{~h} \mathrm{~g}^{-1}$ after 100 cycles when cycled at a current density of $200 \mathrm{~mA} \mathrm{~g}^{-1}$.

Additionally, 2D Si nanostructure possesses a smaller specific variable SEI surface area than that of other dimensional Si nanostructures. From this aspect, Chen et al. [73] synthesized ultrathin mesoporous 2D Si nanosheets by a soft template and subsequent magnesiothermic reduction method. Owing to the ultrathin $2 \mathrm{D}$ characteristics and mesoporous structure, it could simultaneously buffer the structural stress, mitigate the volume expansion, accelerate the electrolyte permeation and shorten the lithium diffusion pathway, giving rise to a superior cycle performance. Moreover, Zhang et al. [74] developed 


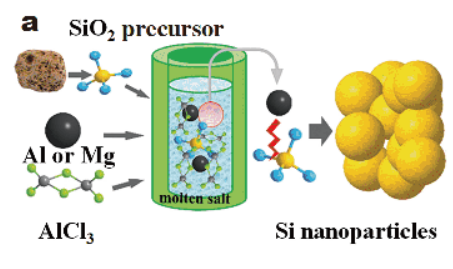

$\mathbf{c}$
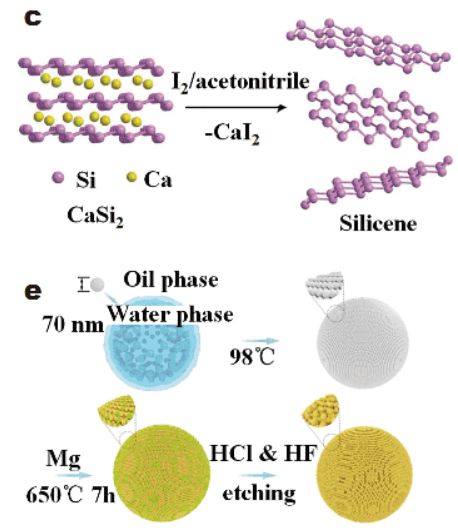
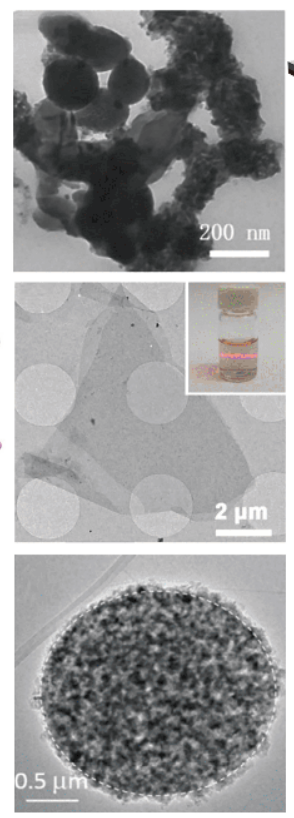

b Si film aCu AgNPs $a \mathrm{Si} / \mathrm{Cu}$

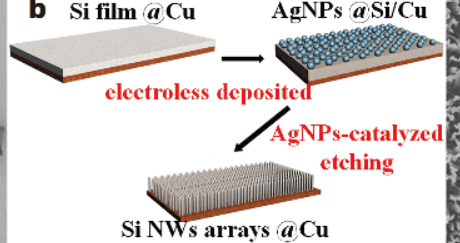

d

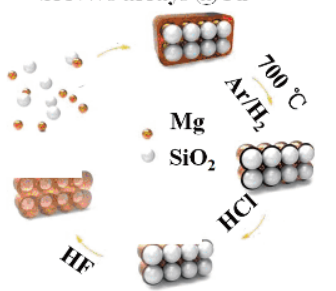

f

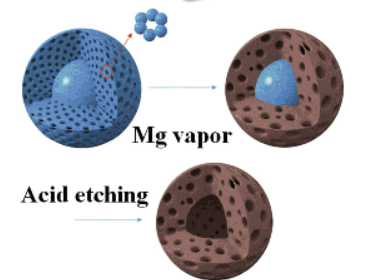

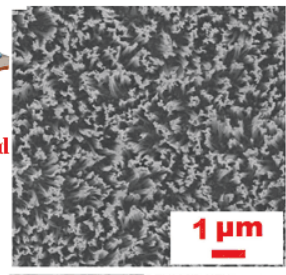
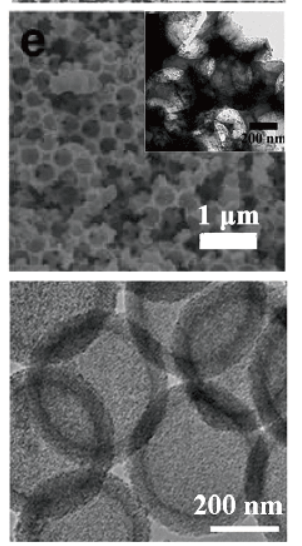

Figure 5 Schematic illustration of the synthesis process of different nanostructured silicons for LIBs: (a) 0D nanoparticles. Reproduced with permission from Ref. [70]. Copyright 2015, the Royal Society of Chemistry. (b) 1D nanowires. Reprinted with permission from Ref. [72]. Copyright 2017, Springer-Verlag GmbH Germany. (c) 2D nanosheets. Reprinted with permission from Ref. [75]. Copyright 2018, Wiley-VCH Verlag GmbH \& Co. (d) 3D hierarchical structures. Reprinted with permission from Ref. [76]. Copyright 2016, American Chemical Society. (e) Porous structure. Reprinted with permission from Ref. [77]. Copyright 2018, Elsevier Ltd. (f) Hollow structures. Reprinted with permission from Ref. [78]. Copyright 2015, Nature Publishing Group.

a dual stabilized silicon building block, comprised of inherently interconnecting, spatially orientated silicene nanoplates to address the structure and interface stability issues of silicon. Lately, a scalable preparation of freestanding high-quality silicene via liquid oxidation and exfoliation of $\mathrm{CaSi}_{2}$ was reported by Liu et al. [75] (Fig. 5c). The obtained silicene with monolayer or fewlayer thickness was further used for LIB's anode, exhibiting a reversible capacity of $721 \mathrm{~mA} \mathrm{~h} \mathrm{~g}^{-1}$ at $0.1 \mathrm{~A} \mathrm{~g}^{-1}$ and an extraordinary cycling stability with no capacity decay after 1800 cycles.

Apart from the downsizing silicon with regulable morphology strategy, creating macro/mesopores silicon structure is another effective way to accommodate distinct volume change that accompanies with the lithiation process. Furthermore, the interconnected pores provide the channels for quick lithium ions transport and full infiltration of electrolyte, which guarantee an excellent rate capability and cycle stability. Well-crystallized 3D hierarchical macro-/mesoporous silicon structure (Fig. 5d) was synthesized through the magnesiothermic reduction process using $\mathrm{OD}$ silicon particles as selftemplate [76]. The macro-/mesoporous silicon exhibited significantly improved cyclic and rate performance. A reversible capacity of $959 \mathrm{~mA} \mathrm{~h} \mathrm{~g}^{-1}$ was retained after 300 cycles at $0.2 \mathrm{Ag}^{-1}$ with a high mass loading density of $1.4 \mathrm{mg} \mathrm{cm}^{-2}$. Consistent with this strategy, Jia et al. [77] synthesized micrometer-sized silicon-based anodes with a unique porous structure (Fig. 5e) demonstrating good electrochemical performance. The void space derived from mesopores by the removal of the $\mathrm{MgO}$ phase could effectively accommodate the volume change of silicon and thus alleviate the breakdown of the SEI layer, all of which led to a high reversible capacity of $1467 \mathrm{~mA} \mathrm{~h} \mathrm{~g}^{-1}$ at $2.6 \mathrm{~A} \mathrm{~g}^{-1}$, good rate capacity, and superior cycling performance up to 370 cycles.

Introduction of space into the nanostructured silicon to deliver an extra room is another efficient way to accommodate the unavoidable volume expansion and buffer the Li-ion diffusion induced mechanical strain. Xiao et al. [78] developed a combination of magnesiothermal reduction and acid etching method for the synthesis of hollow porous Si nanospheres (Fig. 5f). In situ TEM and chemo-mechanical modelling characterization demonstrated that both the mesoporous shell and hollow internal void were responsible for the inward expansion, contraction and stable SEI formation during lithiation and delithiation, which further enhanced the capacity 
retention and cycling performance.

\section{Si-carbon composites}

Combining nanoscale silicon with various morphologies and structures has been proven to be an efficient strategy to improve the LIB performance. Although nanosized silicon can address the pulverization, the volume expansion of silicon still exists, which means that the SEI films will be destroyed and continuously produced. This will consume a large number of lithium ions, seriously reducing the $\mathrm{CE}$ and the reversible specific capacity of batteries. In addition, the conductivity of nano silicon is still an issue to improve the rate performance.

To date, carbon material is considered as one of the best candidates to address the above issues of silicon electrode because of its outstanding electrical conductivity and mechanical strength [79]. Conventional Si-C composites were commonly produced by mechanical mixing method, leading to an inhomogeneous disperse and poor connectivity of each component. In this regard, carbon uniformly coated on silicon with diverse structures becomes a promising approach to improve the electrochemical performance [54,55].

Encapsulation of polybenzimidazole (PBI) derived from pyrrolic $\mathrm{N}$-enriched carbon onto microsized silicon spheres [80] was achieved by an aerosol-assisted assembly combined with physisorption process (Fig. 6a). Thanks to the intrinsic high electronic conductivity, abundant pyrrolic nitrogen and structure robustness, the mesoporous Si-PBI carbon composite can avoid direct contact between silicon and electrolyte, prevent uncontrolled SEI film formation and create numerous extrinsic defects and active sites for extra lithium storage, which further enable the Si-PBI carbon composite with superior electrochemical performance in terms of high reversible specific capacity $\left(2172 \mathrm{~mA} \mathrm{~h} \mathrm{~g}^{-1}\right)$, superior rate capability (1186 $\mathrm{mA} \mathrm{h} \mathrm{g}^{-1}$ at $5 \mathrm{~A} \mathrm{~g}^{-1}$ ) and prolonged cycling life.

In order to buffer the volume expansion effect of silicon material more effectively, introducing voids between silicon and carbon layer is a more effective way [42,81]. In addition, the carbon shell prevents the inner silicon cores from aggregation and assists the formation of a stable SEI layer on the shell surface. Guo et al. [82] fabricated porous silicon nanoparticles ( $\mathrm{p}$-SiNPs) loaded in con-

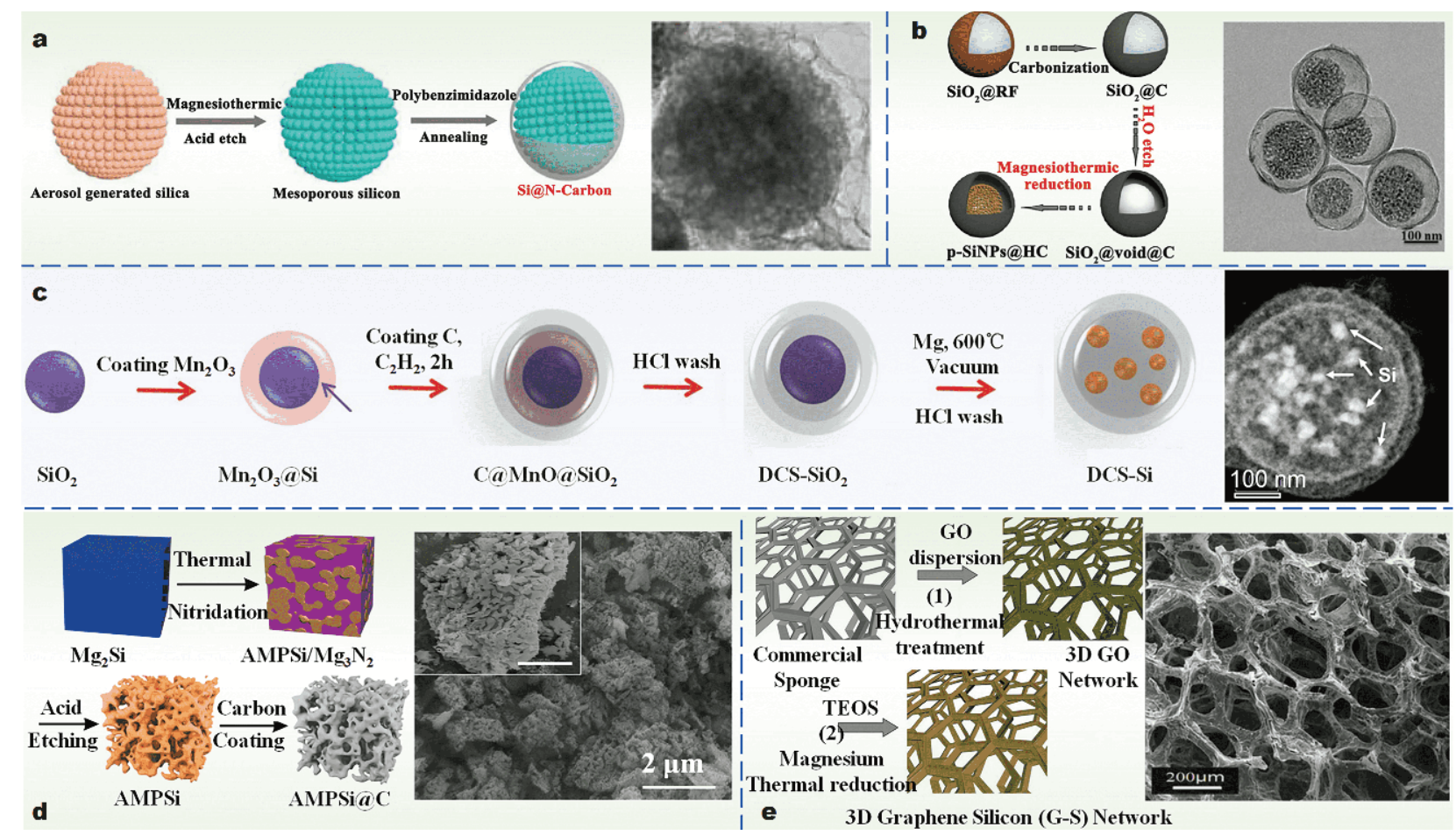

Figure 6 Schematic illustration of the synthesis process of Si-C composites and the corresponding morphologies or microstructures for LIBs: (a) Core-shell Si@N-C. Reproduced with permission from Ref. [80]. Copyright 2017, American Chemical Society. (b) Yolk-shell p-SiNPs@HC. Reproduced with permission from Ref. [82]. Copyright 2017, American Chemical Society. (c) SiNPs@double carbon shells. Reproduced with permission from Ref. [83]. Copyright 2017 Wiley-VCH Verlag GmbH \& Co. (d) Ant-nest-like porous Si-C. Reprinted with permission from Ref. [84]. Copyright 2019, Nature Publishing Group. (e) 3D graphene-Si network. Reproduced with permission from Ref. [85]. Copyright 2015 WILEY-VCH Verlag $\mathrm{GmbH} \& \mathrm{Co}$. 
trollable void carbon spheres with yolk-shell structure by combining hydrothermal water etching and magnesiothermic reduction strategy, as shown in Fig. 6b. The optimized porous silicon nanoparticles@hollow carbon (p-SiNPs@HC) nanohybrids showed excellent LIB performance, delivering a capacity of $1400 \mathrm{~mA} \mathrm{~h} \mathrm{~g}^{-1}$ after 100 cycles at $0.2 \mathrm{~A} \mathrm{~g}^{-1}$ and $720 \mathrm{~mA} \mathrm{~h} \mathrm{~g}^{-1}$ at a high current density of $4 \mathrm{~A} \mathrm{~g}^{-1}$. Moreover, Chen et al. [83] proposed a "double-shell" concept (DCS-Si) to confine the Si volume changes and stabilize the SEI layer using double carbon shells with certain inter spaces by CVD and magnesiothermic reduction method (Fig. 6c). The inner carbon shell provided certain voids to withstand volume changes inside the inner carbon shell and the outer shell stabilized the SEI layer in case of any cracks. Compared with bare Si and single carbon layer coated Si electrodes, the DCS-Si electrode showed a higher specific capacity of $1802 \mathrm{~mA} \mathrm{~h} \mathrm{~g}^{-1}$ at a current rate of $0.2 \mathrm{C}$, superior rate capability and good cycling performance up to 1000 cycles.

Porous structure designs to improve the performance of Si-C composite are similar to those of hollow structures. The space derived from porous structure can endure the volume expansion and alleviate the stresses. In addition, large surface area and uniformly distributed channels shorten the diffusion path for $\mathrm{Li}$ ions and increase reactivity of the composites, thus leading to enhanced rate capability. Lately, An et al. [84] reported a scalable top-down technique by thermal nitridation of the $\mathrm{Mg}-\mathrm{Si}$ alloy and acid etching method to produce ant-nestlike porous silicon (shown in Fig. 6d). Synchrotron radiation tomographic reconstruction images and in-situ TEM characterization reveal that the ant-nest-like porous silicon consisting of 3D interconnected silicon nanoligaments and bicontinuous nanopores could prevent pulverization and accommodate volume expansion during cycling. The carbon-coated porous silicon anode delivered a high capacity of $1271 \mathrm{~mA} \mathrm{~h} \mathrm{~g}^{-1}$ at $2100 \mathrm{~mA} \mathrm{~g}^{-1}$ with $90 \%$ capacity retention after 1000 cycles and had a low electrode swelling of $17.8 \%$ at a high areal capacity of $5.1 \mathrm{~mA} \mathrm{~h} \mathrm{~cm}^{-2}$.

Li et al. [85] reported an efficient approach to facile and large-area fabrication of 3D graphene-silicon (G-Si) networks that possessed porous structure, good flexibility, ultrathin hybrid walls and high electrical conductivity, as displayed in Fig. 6e. All these unique properties are beneficial to minimizing the volume change of silicon and facilitating the diffusions of both lithium and electron. As a consequence, the resulting 3D G-Si network exhibited a superior rate capability, cyclability and high reversible capacity over $2050 \mathrm{~mA} \mathrm{~h} \mathrm{~g}^{-1}$ after 200 cycles.

\section{Si-metal composites}

The electrochemical properties of silicon can also be improved by compounding silicon with other metal materials especially at extremely high rates. On the one hand, metal doping can effectively improve the conductivity of silicon and promote the charge transfer process. On the other hand, the proportion of silicon in materials can be reduced by replacing part of silicon, and thus the volume expansion degree can be reduced. Moreover, the introduced metal can buffer the volume expansion of silicon to improve the electrode structural stability by using its good ductility. And some metals can form alloying products with silicon under heat treatment conditions, which are closely bound to silicon and further act as buffer layer to enhance the structural stability of materials when the volume of silicon expands. Besides, compounding with Li-active metals is also attractive, because they have different potentials for lithiation and delithiation. When $\mathrm{Li}$ is inserted into one component, the other will alleviate the volume change as a buffer. Song et al. [86] delivered a template-free synthesis of highly connected hollow Si-Cu nanotubes for high-rate and durable LIB binder-free anode. $\mathrm{CuO}$ was firstly grown on the copper foam by a thermal oxidation treatment, as illustrated in Fig. 7a. Then, coating amorphous silicon on $\mathrm{CuO}$ nanowires was achieved via a plasma-enhanced CVD technology. Finally, hollow Si-Cu nanotubes were obtained by an in situ $\mathrm{H}_{2}$ annealing. The resulting hollow $\mathrm{Si}$-Cu nanotubes anode possessed a high specific capacity of $780 \mathrm{~mA} \mathrm{~h} \mathrm{~g}^{-1}$ after 1000 cycles at $20 \mathrm{~A} \mathrm{~g}^{-1}$, with a capacity retention rate of $88 \%$.

Recently, 3D nanoporous SiGe alloy with tunable morphology and porosity [87] was synthesized as a highperformance LIB anode using a dealloying method with a ternary AlSiGe ribbon serving as the precursor, as shown in Fig. $7 \mathrm{~b}$. By adjusting the $\mathrm{Al}$ content in the precursor, the morphology and porosity could be controlled. With an $\mathrm{Al}$ content of $80 \%$, the 3D-NP SiGe with continuous ligaments and hierarchical micropores and mesopores coral-like structure delivered a high reversible capacity of $1158 \mathrm{~mA} \mathrm{~h} \mathrm{~g}^{-1}$ after 150 cycles at a current density of $1000 \mathrm{~mA} \mathrm{~g}^{-1}$ with excellent rate capacity.

In addition, according to different preparation conditions and design methods, silicon/metal composites with different structures can be obtained. Zhang et al. [88] developed a composite anode of $\mathrm{Cu} / \mathrm{Si} / \mathrm{Ge}$ nanowire arrays, where each nanowire consisted of a core of $\mathrm{Cu}$ segments and a Si/Ge bilayer shell, as illustrated in Fig. 7c. 

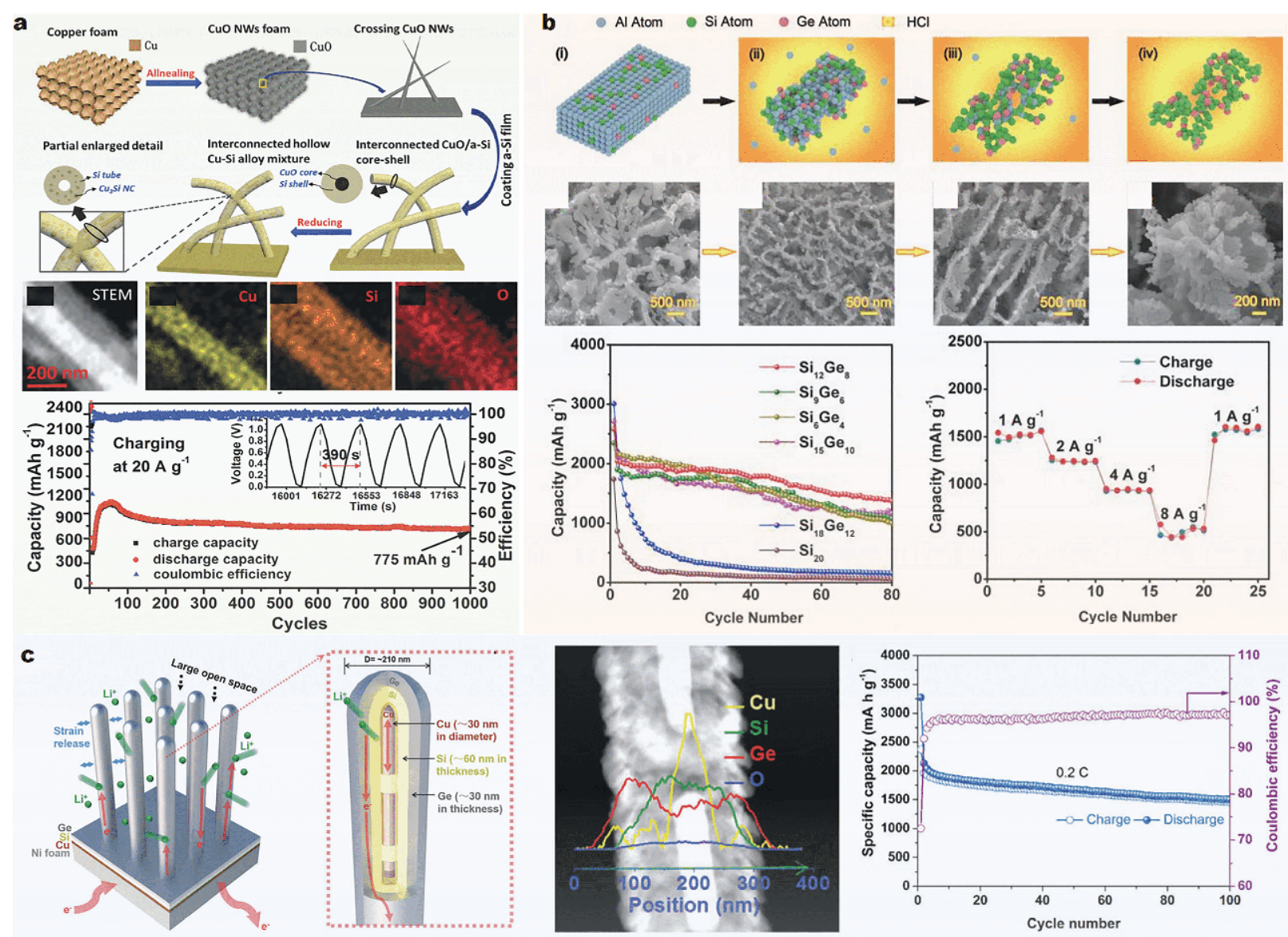

Figure 7 Si-metal composites. (a) Schematic illustration of the synthesis process of $\mathrm{Cu}$-Si alloy structures, scanning transmission electron microscopy (STEM), energy-dispersive X-ray spectroscopy (EDS) mapping of CuO/amorphous-Si core-shell structure and the long term cycling performance at $20 \mathrm{~A} \mathrm{~g}^{-1}$. Repinted with permission from Ref. [86]. Copyright 2015, WILEY-VCH Verlag GmbH \& Co. (b) Schematic illustration and SEM of the 3DNP SiGe structure evolution via chemical dealloying, Cycle performance of the 3D-NP SiGe anodes and Si20 anode at $100 \mathrm{~mA} \mathrm{~g}$ and rate capability of the $\mathrm{Si}_{12} \mathrm{Ge}_{8}$ anode at different current densities from 1 to $8 \mathrm{~A} \mathrm{~g}^{-1}$. Reprinted with permission from Ref. [87]. Copyright 2018, American Chemical Society. (c) Schematic showing of $\mathrm{Cu} / \mathrm{Si} / \mathrm{Ge}$ array grown on a Ni foam substrate, STEM image of a $\mathrm{Cu} / \mathrm{Si} / \mathrm{Ge}$ nanowire with corresponding EDS spectra and capacity performance at a low rate of 0.2 C. Repinted with permission from Ref. [88]. Copyright 2018, the Royal Society of Chemistry.

This unique electrode architecture exhibited several favorable properties: the nanoscale nanowires enabled facile strain relaxation; the free space between nanowires and the hollow space between $\mathrm{Cu}$ segments accommodated volume expansion; the core $\mathrm{Cu}$ segments enhanced electron transport. Moreover, the outer Ge shell also served as an active high-capacity coating. By using in-situ TEM and electrochemical testing, a novel co-lithiation/co-delithiation reaction mechanism was proposed, which effectively alleviated the electrochemically induced mechanical degradation and thus greatly enhanced the long-cycle stability of the electrode.

\section{Si-metal oxide composites}

Unlike alloys, Si/metal oxide composites with a core-shell structure share a similar working mechanism with $\mathrm{Si} / \mathrm{C}$ core-shell composites. The metal oxide coating layer on $\mathrm{Si}$ can separate silicon from electrolyte and stabilize SEI film. To add on this concept, Fang et al. [89] reported a rational design of core-shell $\mathrm{Si}_{2} \mathrm{TiO}_{2}$ with Si NPs encapsulated in $\mathrm{TiO}_{2}$ hollow spheres by combination of hydrolysis with magnesiothermic reduction method, as shown in Fig. 8a. In this architecture, the void space inside provided enough space for accommodation of the volume expansion, and the robust $\mathrm{TiO}_{2}$ shell could improve the electrical conductivity and prevent the direct contact of $\mathrm{Si}$ with the electrolyte. As a result, the $\mathrm{Si@TiO}{ }_{2}$ hollow nanospheres maintained a specific capacity of $804 \mathrm{~mA} \mathrm{~h} \mathrm{~g}^{-1}$ after 100 cycles at $0.1 \mathrm{C}$.

Lately, a facile sol-gel strategy was proposed to syn- 

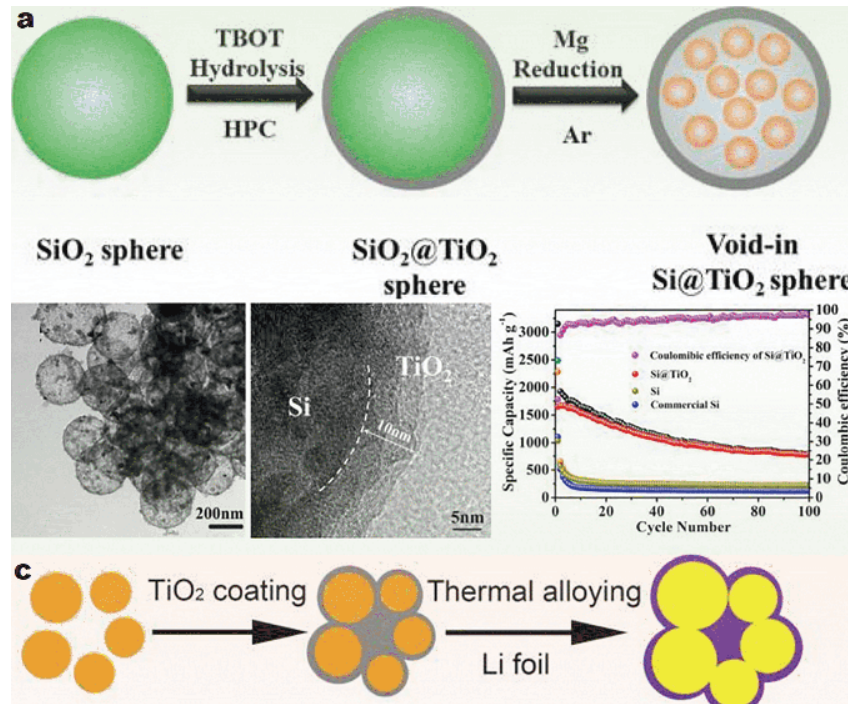

Li foil
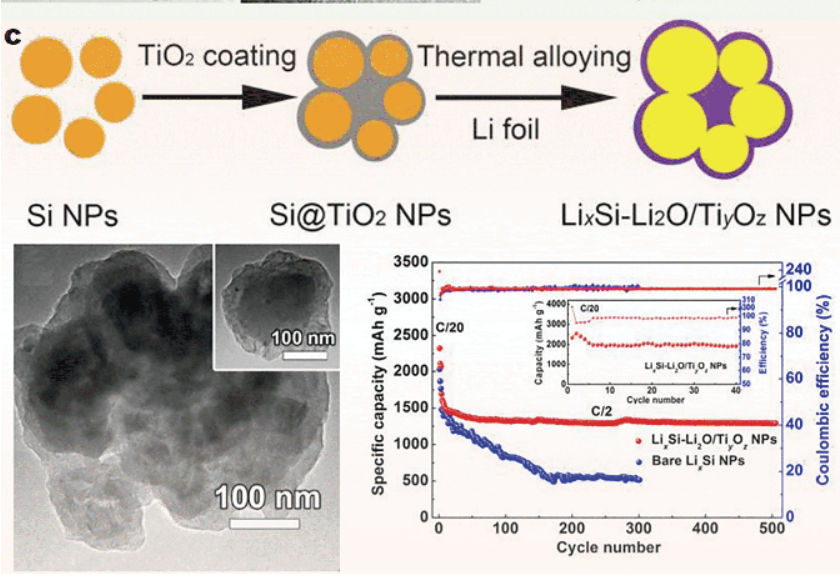

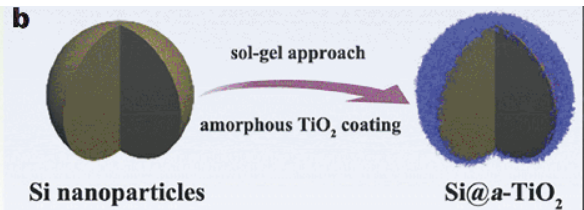

$\mathrm{Si}$ a- $\mathrm{TiO}_{2}$
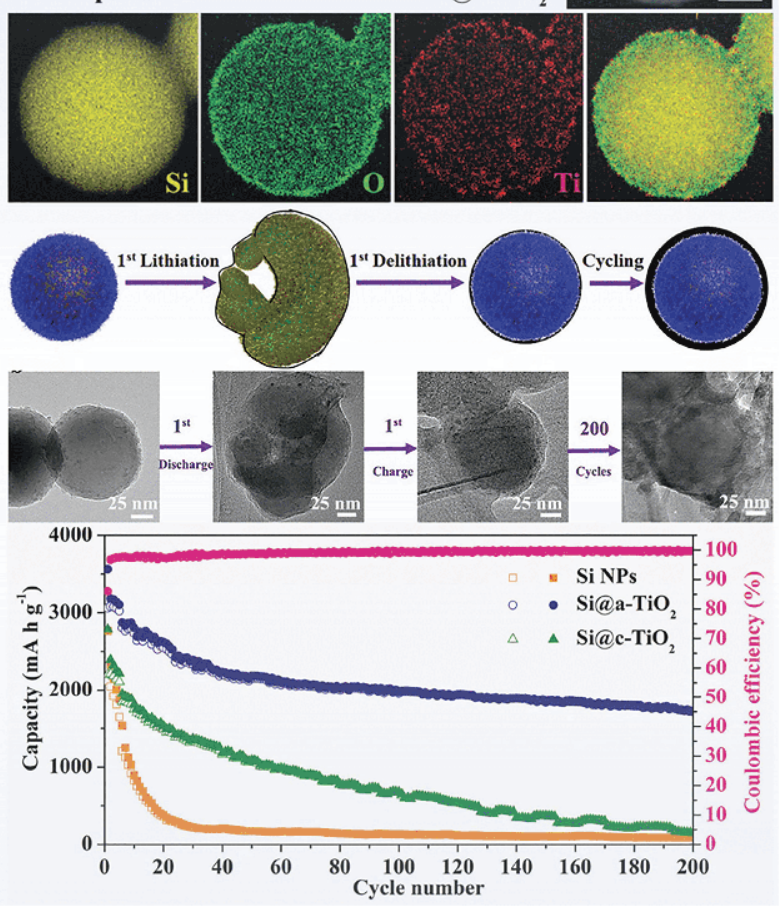

Figure 8 Metal oxides coating on silicon. (a) Schematic illustration for the fabrication process of the unique core-shell nanostructure of the Si@TiO 2

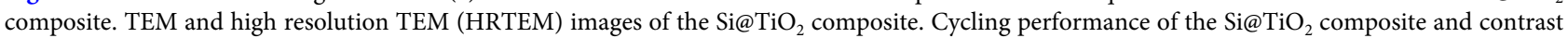
samples at 0.1 C. Reproduced with permission from Ref. [89]. Copyright 2014, American Chemical Society. (b) Schematic of fabrication process of the amorphous- $\mathrm{TiO}_{2}$-coated $\mathrm{Si}$ core-shell nanoparticles, STEM image and the corresponding EDS mapping images, schematic and TEM images of structural evolution of the amorphous- $\mathrm{TiO}_{2}$-coated commercial Si nanoparticle electrode during electrochemical cycling, and cycling performance of the Si, Si@a-TiO ${ }_{2}$, and Si@crystal- $\mathrm{TiO}_{2}\left(\mathrm{c}-\mathrm{TiO}_{2}\right)$ nanoparticle electrodes. Reprinted with permission from Ref. [90]. Copyright 2017, WILEY-VCH Verlag GmbH \& Co. (c) Schematic of the fabrication process for $\mathrm{Li}_{x} \mathrm{Si}_{-}-\mathrm{Li}_{2} \mathrm{O} / \mathrm{Ti}_{y} \mathrm{O}_{z}$ core-shell NPs, TEM images of the resultant $\mathrm{Li}_{x} \mathrm{Si}_{-}-\mathrm{Li}_{2} \mathrm{O} / \mathrm{Ti}_{y} \mathrm{O}_{z} \mathrm{NPs}$ and cycling performances of $\mathrm{Li}_{x} \mathrm{Si}-\mathrm{Li}_{2} \mathrm{O} / \mathrm{Ti}_{y} \mathrm{O}_{z}$ core-shell NPs and bare $\mathrm{Li}_{x} \mathrm{Si}$ NPs. Reprinted with permission from Ref. [91]. Copyright 2018, American Chemical Society.

thesize core-shell Si@amorphous $\mathrm{TiO}_{2}(\sim 3 \mathrm{~nm})$ by Yang et al. [90], as exhibited in Fig. 8b. STEM and the elemental mapping characterizations clearly verified that a thin $\mathrm{TiO}_{2}$ nanograin layer was conformally coated on the Si nanoparticle core. Ex situ TEM measurements revealed the structural evolution of the core-shell Si@amorphous$\mathrm{TiO}_{2}\left(\mathrm{a}-\mathrm{TiO}_{2}\right)$ nanoparticles during the initial lithiation and delithiation processes. The $\mathrm{a}-\mathrm{TiO}_{2}$ shell showed elastic behavior during cycling, avoided the electrolyte impregnation within the encapsulated Si core and finally maintained the structural integrity. Further electrochemical investigation revealed that the $\mathrm{a}-\mathrm{TiO}_{2}$ shell offered superior buffering properties over the pristine $\mathrm{Si}$ nanoparticles and crystalline $\mathrm{TiO}_{2}$ layers coated $\mathrm{Si}$ for unprecedented cycling stability. More recently, Wang et al. [91] reported coating $\mathrm{Si}$ with a- $\mathrm{TiO}_{2}$ layers and then thermally alloy lithiation approach to prepare lithiated$\mathrm{TiO}_{2}$ protected $\mathrm{Li}_{x} \mathrm{Si}$ nanoparticles $\left(\mathrm{Li}_{x} \mathrm{Si}-\mathrm{Li}_{2} \mathrm{O} / \mathrm{Ti}_{y} \mathrm{O}_{z}\right.$ $\mathrm{NPs}$ ) as a LIB anode (displayed in Fig. 8c). The robust lithiated- $\mathrm{TiO}_{2}$ matrix not only improved the electrical conductivity but also spatially limited the direct SEI formation on $\mathrm{Li}_{x} \mathrm{Si} / \mathrm{Si}$ cores during cycling. More importantly, the coating layer protected the most inner $\mathrm{Li}_{x} \mathrm{Si}$ alloys from corrosion, leading to high dry-air stability. As a result, the resulting $\mathrm{Li}_{x} \mathrm{Si}-\mathrm{Li}_{2} \mathrm{O} / \mathrm{Ti}_{y} \mathrm{O}_{z}$ anode achieved a capacity of about $1300 \mathrm{~mA} \mathrm{~h} \mathrm{~g}^{-1}$ after 500 cycles at a high current rate of $0.5 \mathrm{C}$.

\section{Si-conductive polymer composites and binders}

Besides the carbonaceous, metal and metal oxide mate- 
rials, conducting polymers with unique features including excellent chemical stability, superior electronic conductivity and structural flexibility have also been introduced to accommodate the volume expansion of $\mathrm{Si}$ and to form conductive networks for enhanced reaction kinetics of the electrode. A strong interface bond between $\mathrm{Si}$ and polymer and a high electrical conductivity of polymer are the most critical parameters determining the electrochemical performance of $\mathrm{Si} /$ conducting polymer composite electrodes.

For instance, strong interfacial bond between polyaniline (PANi) and Si NPs was achieved through hydrogen bonding with the native oxide layer on Si particles by insitu polymerization [92], as diaplayed in Fig. 9a. The resulting materials were in a well-connected electrically conductive 3D network consisting of Si NPs conformally coated by the conducting polymer, whose porous space was beneficial to volume expansion of Si particles. In contrast to fast capacity fading for Si/PANi mixture, an electrode capacity of $550 \mathrm{~mA} \mathrm{~h} \mathrm{~g}^{-1}$ was still retained after 5000 cycles with over $90 \%$ capacity retention at current density of $6.0 \mathrm{~A} \mathrm{~g}^{-1}$ for the in-situ polymerized Si/PANi electrodes. Du et al. [93] reported the synthesis of PPy@porous silicon hollow spheres (PPy@PHSi) via the magnesiothermic reduction of mesoporous silica hollow nanospheres $\left(\mathrm{MHSiO}_{2}\right)$ and in situ chemical polymerization of PPy on the PHSi surface (shown in Fig. 9b). The synergetic effect of the porous hollow structure and the surface PPy coating is responsible for the highly electrochemical characteristics. The hollow structure and porous channels in the shell can not only buffer the huge volume change and reduce the diffusion-induced stress, but also facilitate the diffusion of $\mathrm{Li}^{+}$and electrolyte into the electrode. The surface PPy coating can significantly enhance the surface electronic conductivity and stabilize the whole structure. As a part of the electrode, polymer

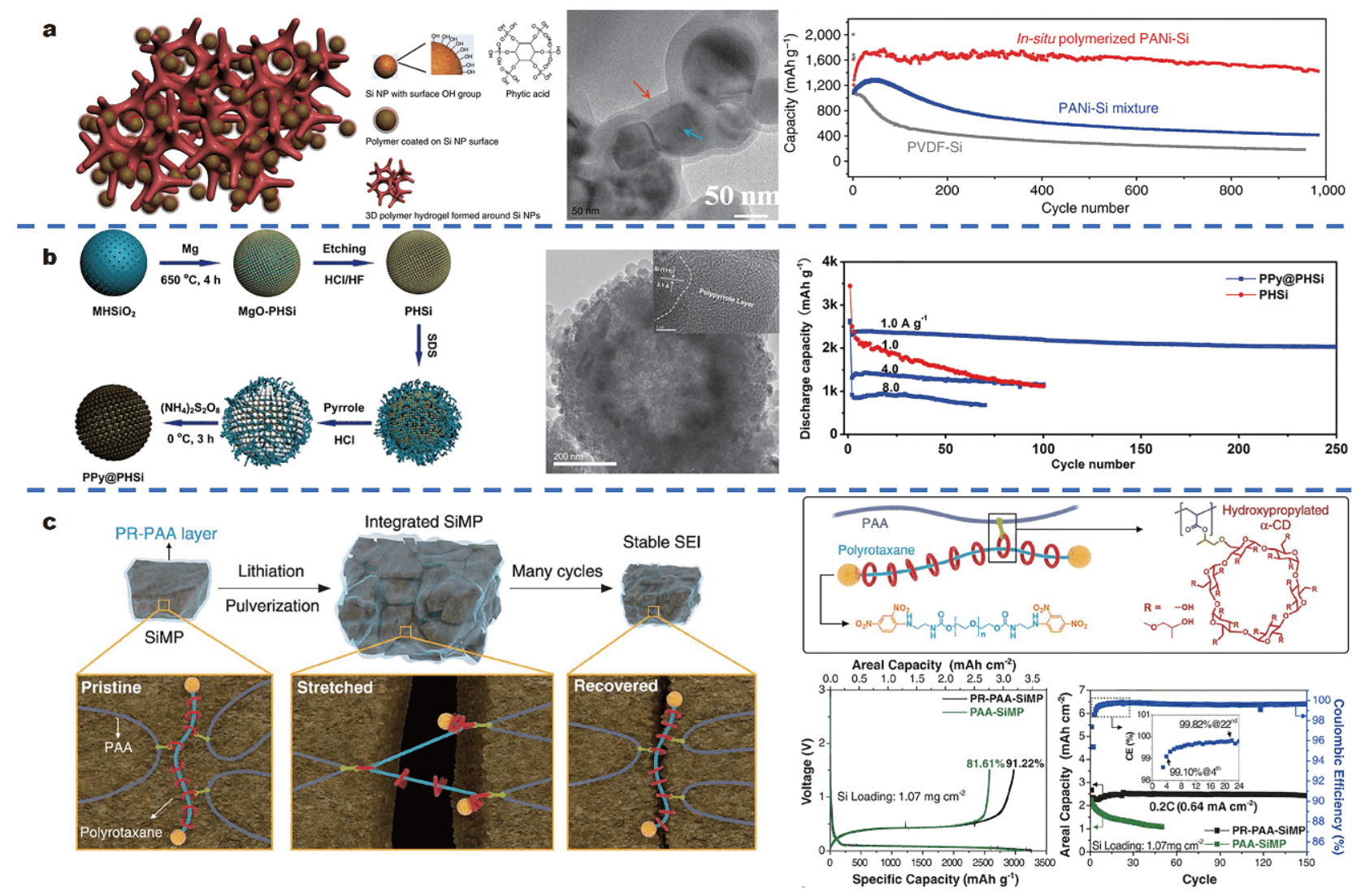

Figure 9 (a) Schematic illustration of the 3D porous PANi-Si electrodes, TEM of the SiNPs coated with PANi polymer layer and cycling performance of the PANi-Si composite electrode and contrast materials at current of $1.0 \mathrm{~A} \mathrm{~g}^{-1}$. Reproduced with permission from Ref. [92]. Copyright 2013, Nature Publishing Group. (b) Schematic of the fabrication process of PPy@PHSi nanocomposite, TEM and cycling performance of the PHSi and PPy@PHSi nanocomposite at a variety of current densities. Reprinted with permission from Ref. [93]. Copyright 2014, WILEY-VCH Verlag GmbH \& Co. (c) Graphical showing of the operation mechanism of PR-PAA during repeated volume changes of SiMPs, together with chemical structures of polyrotaxane and PAA and the corresponding electrochemical performance. Reproduced with permission from Ref. [95]. Copyright 2017, AAAS. 
binders act as bonds between current collectors, active substances and conductive agents to ensure electrical contact and integrity of electrodes. Strong supramolecular interactions such as hydrogen bond, ion-dipole interaction and coordination bond play an important role in guiding the structural design of binders. Sodium alginate was firstly used as a binder for silica-based materials [94]. The polar functional groups in sodium alginate molecules could form hydrogen bonds with the silica layer on the silicon surface, which could effectively maintain the bonding between sodium alginate binder and silica materials and the integrity of the electrode structure. In addition, sodium alginate could maintain high strength even in electrolyte. Therefore, it could effectively keep a stable conductive network and buffer the volume change of silicon, thus improving the cycling performance of the electrode.

Recently, Choi et al. [95] combined the advantages of soft elastic material polyacrylic acid (PAA) and rigid plastic polyrotane (PR) for highly efficient binders (displayed in Fig. 9c). The soft elastic component can ensure the ionic and electronic conductance of active material and even keep the pulverized silicon coalesced without disintegration, enabling stable cycle life for silicon microparticle in the process of cycling. Noteworthily, the conducting polymers also could be substitution for traditional conductive additive or binder as a conductive binder which reduced the nonactive components [96]. For example, Zeng et al. [97] designed and prepared a novel polymer binder possessing high ion and electron conductivities by assembling ion-conductive polyethylene oxide (PEO) and polyethylenimine (PEI) onto the electron-conductive poly(3,4-ethylenedioxythiophene): poly(styrenesulfonate) (PEDOT:PSS) chains via chemical crosslinking, chemical reduction and electrostatic self-assembly. The obtained polymer binder possessed superior 14 and 90 times higher of lithium-ion and electron transport properties than those of carboxymethyl cellulose (CMC) (with acetylene black) binder system. What's more, the strong crosslinking and electrostatic interactions guaranteed the integrity of the $\mathrm{Si}$ anode during volume contraction. Consequently, the Si anode with the final conductive polymer binder showed enhanced delithiation rate capability, cycling stability, reversible capacity, and initial CE.

Apart from the above direct interactions between the silicon and the binder that could enhance the electrochemical performance of silicon anode, an all-integrated electrode was achieved by Liu et al. [98] with indirect chemical interlinking between carbon shells of car- bon@void@silica@silicon (CVSS) and the polymer binder. Based on density functional theory (DFT) calculations, the binding energy between the binder and the carbon layer of CVSS was higher than that of the direct interactions between the silicon and the binder, which led to a better electrode stability and higher electrochemical performance (a reversible capacity of $1640 \mathrm{~mA} \mathrm{~h} \mathrm{~g}^{-1}$ after 100 cycles at a current density of $1 \mathrm{~A} \mathrm{~g}^{-1}$, and a specifc capacity of $720 \mathrm{~mA} \mathrm{~h} \mathrm{~g}^{-1}$ can be maintained after 1000 cycles at $5 \mathrm{~A} \mathrm{~g}^{-1}$ ).

\section{IN SITU TECHNIQUES FOR FAILURE MECHANISM ANALYSIS}

Promoting the LIB performance of silicon-based anode requires improved understanding of the internal electrochemical processes and the degradation mechanisms in the electrodes. Thus, various in-situ characterization tools have been playing indispensable roles in monitoring the material morphological change, phase evolution, SEI formation and evolution and mechanical property, etc., under real operating conditions. The obtained information is greatly beneficial to the optimization and design of more efficient and stable materials, which in turn improves the overall battery performance. Fundamental understanding and progress revealed by in-situ characterization techniques are discussed in the following section.

\section{Atomic force microscopy}

The lifetime of silicon-based electrodes is closely related to the formation and evolution of the SEI. These passivation films undergo substantial formation when the silicon electrode particles expand and crack during cycling. Thus, study of the formation mechanisms and structural evolution of SEI layers during cycles is of great importance. In-situ AFM has been an invaluable tool to probe the SEI, which can avoid unwanted damage in the sample preparation process such as washing and cleaning. Becker et al. [117] analyzed the shape evolution of a-Si pillars with $100 \mathrm{~nm}$ height and $100-1000 \mathrm{~nm}$ in diameter during cyclings by AFM, as exhibited in Fig. 10a. It was found that the pillars with diameters of $200 \mathrm{~nm}$ or larger reached the theoretical volume expansion of $\sim 300 \%$ and the volume expansion of the $100 \mathrm{~nm}$ pillars was limited. Additionally, the volume effect and interface properties were correlated to the dimension of the materials. Liu et al. [118] investigated the morphology and Young's modulus of the individual Si nanowire during SEI evolution and the electrode volume changes during the first discharging/charging cycle via in-situ AFM (Fig. 10b). 
a
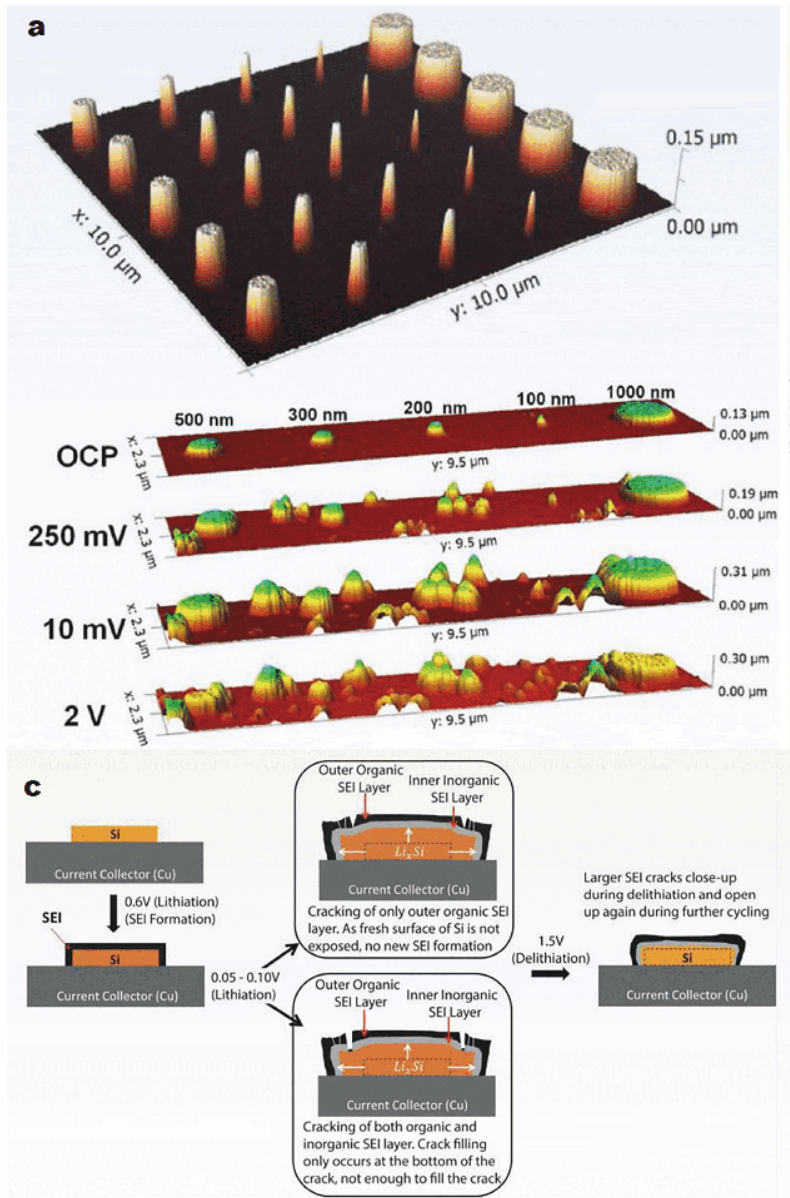

b
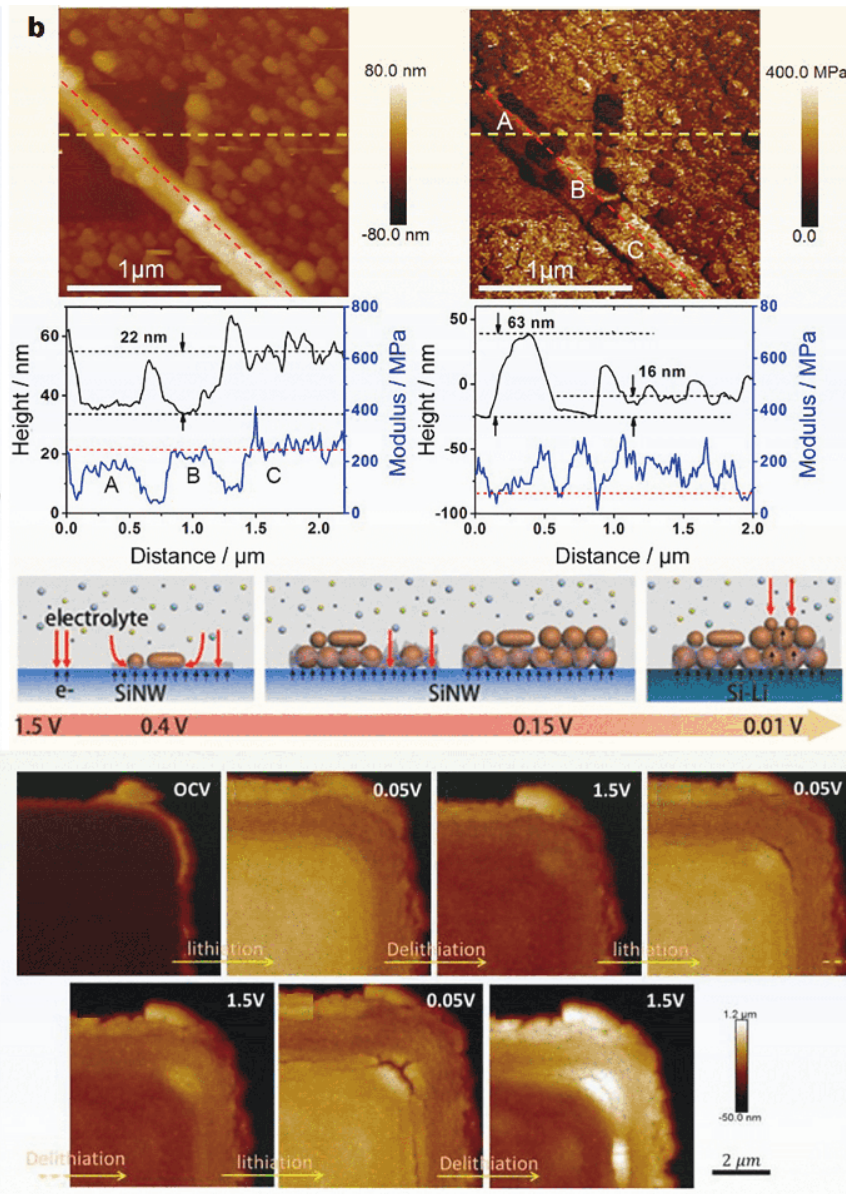

Figure 10 (a) AFM image of the as-fabricated array with a diameter of 1000, 500, 300, 200 and $100 \mathrm{~nm}$ pillars and in situ 3D AFM images during lithiation and delithiation of a-Si nanopillars at several electrochemical potentials in the first cycle. Reproduced with permission from Ref. [117]. Copyright 2013, American Chemical Society. (b) Images of height and modulus of the Si nanowire anode after being scraped. The line profiles of the topography (black) and modulus (blue) along the Si nanowire (marked with a dashed red line) and along the horizontal cross section (marked with a dashed yellow line). Reproduced with permission from Ref. [118]. Copyright 2014, American Chemical Society. (c) Schematic showing the structure evolution of patterned Si island during cycling and the resulting impact of volume changes on SEI formation and failure, in-situ AFM images showing opening and closing of SEI cracks that formed during the initial three cycles. Reproduced with permission from Ref. [119]. Copyright 2016, American Chemical Society.

Three distinct stages of the SEI formation were observed in the discharge process: thin primary film was slowly formed when voltage was above 0.4 ; thick and particlelike SEI grew rapidly when the voltage was in the 0.4-0.1 V range; the SEI continued growing with the voltage below $0.1 \mathrm{~V}$ finally with a thickness of $28 \pm$ $10 \mathrm{~nm}$. The Young's modulus of the Si nanowire interface slightly increased with the growth of SEI. And the final SEI showed a Young's modulus value within a range of 50-400 MPa, consistent with the composition nature of the SEI.

Kumar et al. [119] investigated the mechanical degradation of the extremely fragile SEI layer by inducing controlled strains through the unique Peak Force tapping mode, as shown in Fig. 10c, which directly showed how the volume expansion and contraction of Si electrodes led to significant mechanical damage of the SEI layer. It was found that SEI film in the shear lag region underwent tensile strains during lithiation and the surface cracks did not fill up with new decomposition products in the further cycling.

\section{Transmission electron microscopy}

In-situ TEM is another powerful tool to monitor realtime silicon involved electrochemical reactions including phase evolution, anisotropic expansion and fracture behavior, atomic level details of the lithiation of crystalline $\mathrm{Si}$, effects of coatings and mechanical properties of lithi- 
ated Si $[69,81,120-122]$.

He et al. [123] used in-situ TEM to probe the structural and chemical evolution of molecular layer deposition (MLD) alucone-coated silicon upon cyclic lithiation and delithiation. The native oxide layer ( $2 \mathrm{~nm}$ in thickness) reacted with $\mathrm{Li}$ to form crystalline $\mathrm{Li}_{2} \mathrm{O}\left(\mathrm{c}-\mathrm{Li}_{2} \mathrm{O}\right)$ upon initial lithiation, which further insulated the particle during subsequent cycles. In comparison, the alucone MLD coated silicon showed no agglomeration tendency and $\mathrm{Li}_{2} \mathrm{O}$ formation, and appeared to possess great flexibility, compatible stretching or shrinking ability along with the expansion or shrinkage of the silicon nanoparticles during cycling (shown in Fig. 11a). Si nanoparticle (NP) confined in CNT was applied as a model to investigate the structural change of the Si NPs and the confinement effect of the CNT during the lithiation and delithiation processes by in-situ TEM [124]. It was found that the volume expansion $(\sim 180 \%)$ of the lithiated $\mathrm{Si}$ NPs was restricted by the wall of the CNTs and that the CNT could accommodate this volume expansion without breaking its tubular structure (displayed in Fig. 11b). The Si NP-filled CNTs showed a high reversible lithium sto-
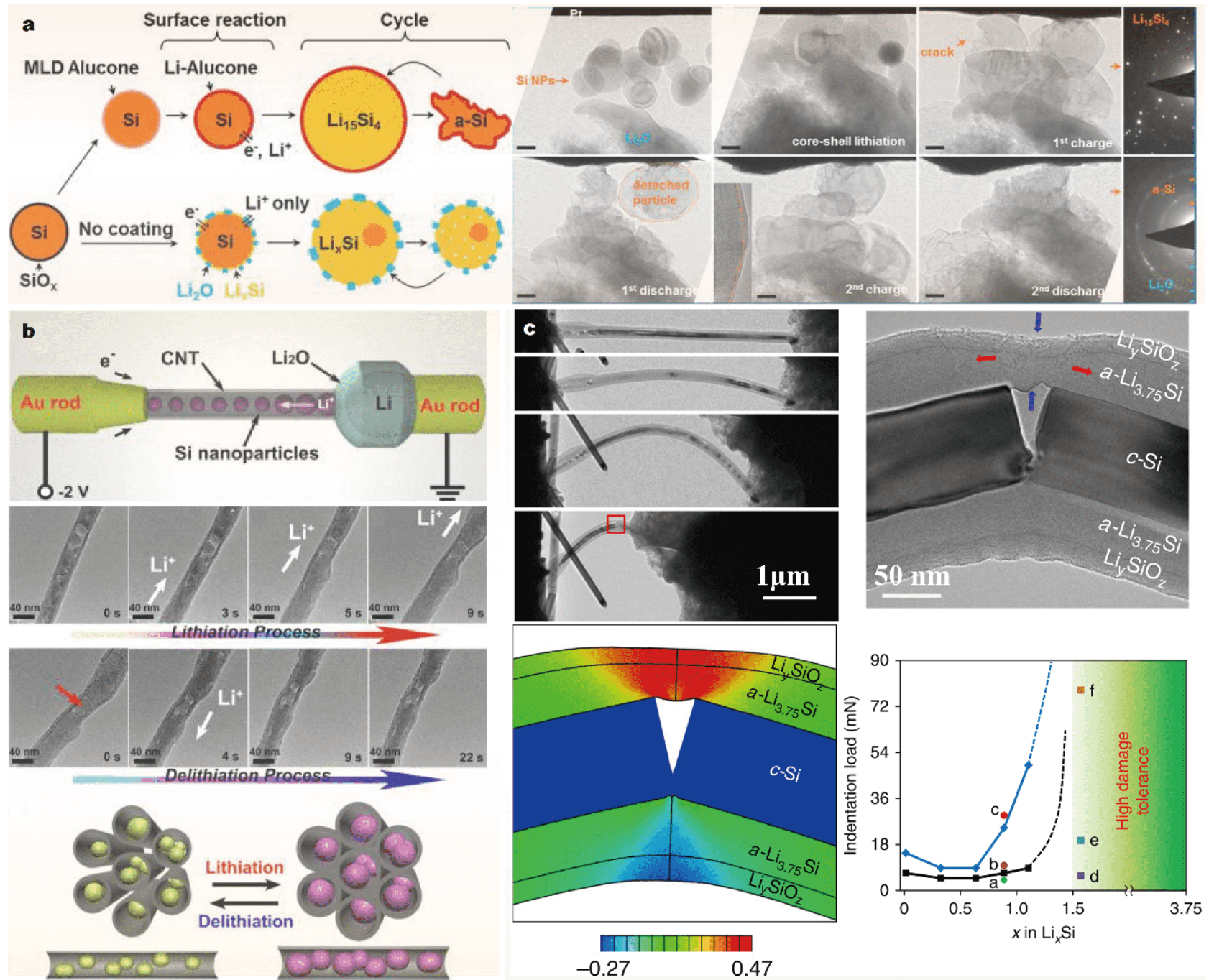

Figure 11 (a) Schematic of surface reactions and cycle behaviors of silicon nanoparticles with different coating conditions and captured in situ TEM images of the alucone-coated Si nanoparticles during the lithiation/delithiation behavior. Reproduced with permission from Ref. [123]. Copyright 2014, American Chemical Society. (b) Schematic of the construction of the electrochemical testing setup, in-situ captured images of a Si NP-filled CNT structure evolution during lithiation and delithiation. Reproduced with permission from Ref. [124]. Copyright 2015, American Chemical Society. (c) Sequential and zoom-in TEM images of the partially lithiated Si nanowire under axial compression, finite element result showing the simulated elastic-plastic deformation in the nanowire that agrees with the zoom-in TEM image and the indentation loads applied to the lithiated electrodes with different Li contents. Reproduced with permission from Ref. [125]. Copyright 2015, Nature Publishing Group. 
rage capacity and desirable high rate capability, because the pulverization and exfoliation of the Si NPs confined in CNTs were efficiently prevented. The fracture resistance is a critical parameter to indicate the structural durability of Si electrode during cycles. Wang et al. [125] reported an in-situ TEM study on the damage tolerance of electrochemically lithiated silicon (exhibited in Fig. 11c). The results revealed a striking contrast of brittle fracture in pristine silicon versus ductile tensile deformation in fully lithiated silicon. The nano indentation testing of amorphous lithiated Si alloys indicated a drastic increase of fracture toughness as the $\mathrm{Li}$ to $\mathrm{Si}$ ratio was increased to above 1.5 .

\section{Other in-situ techniques}

There are other in-situ techniques focusing on studying different aspects of Si-based electrode. For instance, Ogata et al. [126] used operando 7Li NMR spectroscopy to study the kinetics of the electrochemical lithiation and delithiation reactions that occured in $\mathrm{nm}$-sized Si based anodes under realistic cycling conditions (with careful voltage/current controls) over multiple cycles. The ability to control the voltage and current carefully (Fig. 12c) enabled an understanding of how the charge and discharge process in the NMR experiment were connected, for example, the inhomogeneous growth of crystalline $\mathrm{Li}_{3.75} \mathrm{Si}$ from the amorphous phase, the electrochemical and NMR signatures of the process involving the overlithiation of $\mathrm{c}-\mathrm{Li}_{3.75} \mathrm{Si}$ that occured below $50 \mathrm{mV}$, and the formation of small clusters within the $\mathrm{Li}_{3.75} \mathrm{Si}$ phase on charge. These results, in combination with DFT calculations, showed that the phases formed during delithiation strongly depended on the rate of lithiation.

In-situ XRD technique has been applied to study the phase evolution of $\mathrm{c}$-Si electrodes during lithiation/delithiation cycle (Fig. 13a, b). It was found that the formation of metastable c- $\mathrm{Li}_{15} \mathrm{Si}_{4}$ phase in $\mathrm{Si} \mathrm{NW}$ electrodes could be avoided through devising several modifications to materials and processing parameters by limiting the cycles to a lower specific capacity cutoff or altering the SiNW growth temperature [127].

Recently, Operando Raman and synchrotron XRD were combined to probe the evolution of stress and elastic strain in SiNPs anodes during the first two cycles, under
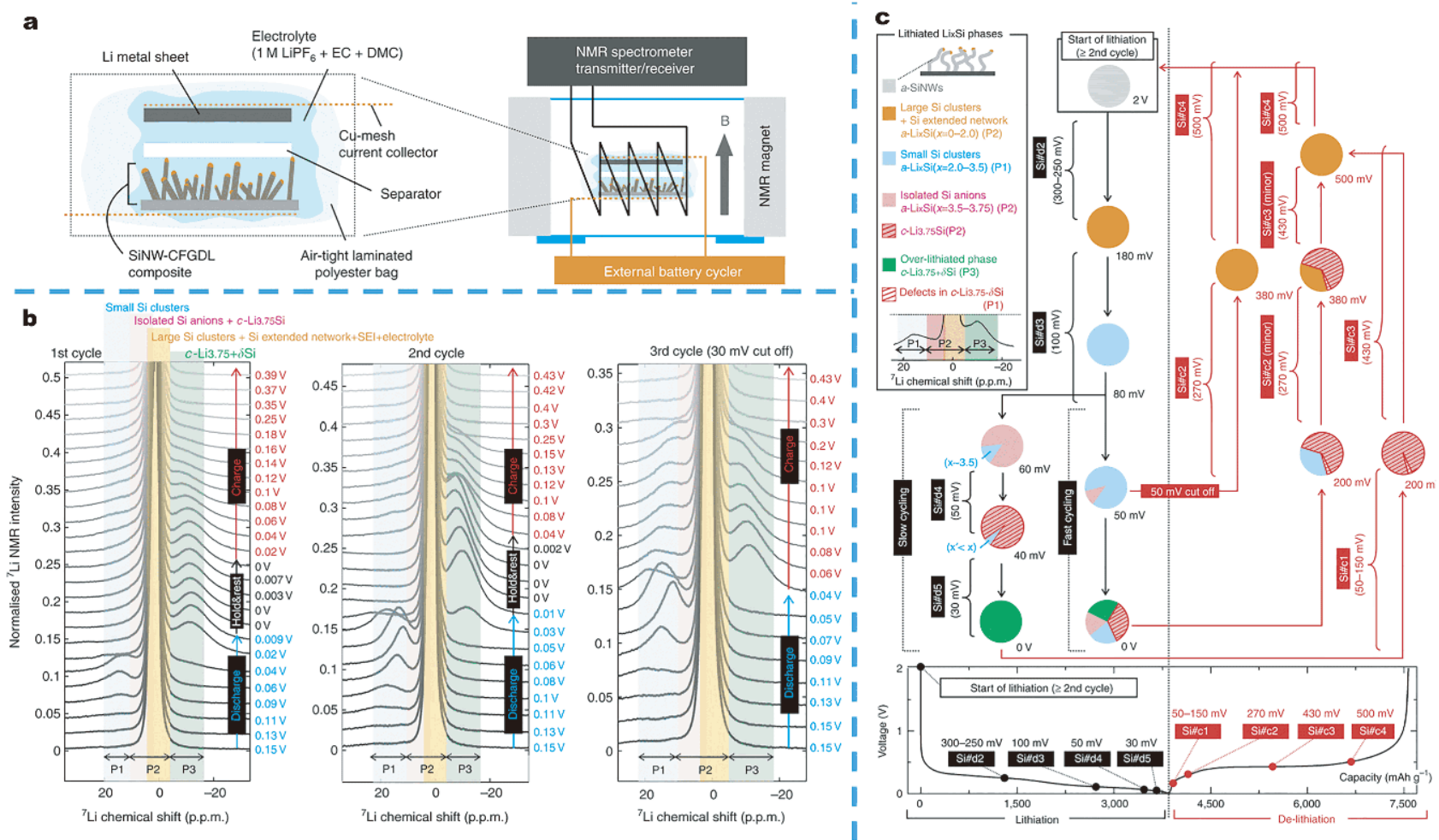

Figure 12 (a) Schematics of the Si nanowire-composite based cell for the in situ 7Li NMR measurements. (b) Enlarged in-situ 7Li NMR spectra of the $\mathrm{Si}$ nanowire-CFGDL composite obtained during the potentiostatic experiment in the first three cycles. (c) Phase transformation diagram for the amorphous silicon nanowires on lithiation and delithiation showing the dependence of the phase evolutions on the rate of cycling. Reproduced with permission from Ref. [126]. Copyright 2014, Nature Publishing Group. 

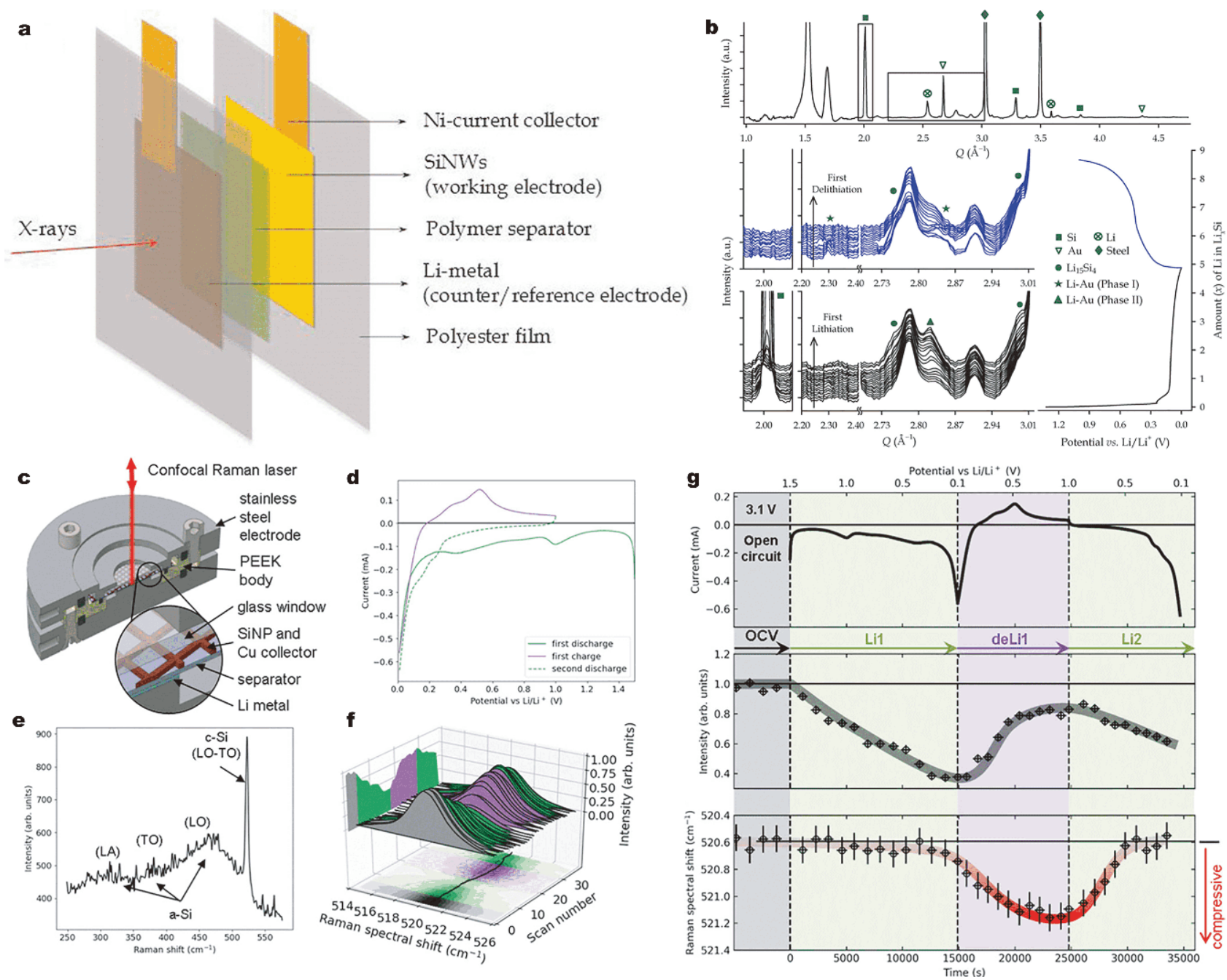

Figure 13 (a) Schematic figure of a cell for in-situ XRD test. (b) In situ XRD results for a stainless steel-mesh (SS-mesh) cell cycled at 0.2 C. Reproduced with permission from Ref. [127]. Copyright 2012, American Chemical Society. (c) Operando Raman setup and electrochemical cell. (d) Linear sweep cyclic voltammograms. (e) Typical Raman spectra including crystalline and amorphous silicon peaks. (f) Evolution of the c-Si transverse optical-longitudinal optical (TO-LO) peak as a function of the scan number during the open-circuit, discharge (green) and charge (purple) cycles. The shadow on the left panel shows the variation of the Raman peak intensity over cycling time and the intensity map on the lower panel indicates the shift of the peak position (highlighted by the black line). (g) Evolution of the current, Raman peak intensity and spectral position as a function of time and applied voltage. Reproduced with permission from Ref. [128]. Copyright 2017, American Chemical Society.

limited capacity cycling conditions [128]. Fig. 13g presents the detailed evolution of the current, Raman intensity and position with the applied voltage, which proved the crystalline SiNPs experienced little to no stress during the first lithiation and were compressed during the following delithiation. Moreover, by analyzing the variations of the Si XRD and Raman peaks, the pressure exerted from amorphized shell onto the continuously shrinking crystalline core was responsible for electrochemically driven variations of the internal stress. Lithiation/delithiation of the silicon under limited capacity conditions triggered the formation of "crystalline core- amorphous shell" particles, which evidenced as a stepwise decrease in core size, as well as sequences of compressive/ tensile strain due to the stress applied by the shell (Fig. 13c-f).

These conclusions from various in-situ technologies are much needed for the benchmarking of theoretical models and for the further rational design of SiNP-based electrodes.

\section{THE GAP BETWEEN CURRENT STUDY AND REAL APPLICATION}

After years of research and development, although great 
improvements of the silicon-based anodes have been achieved in lab scale, the wide application of the Si-based anode in real batteries has not yet popularized. There are only a few reports about the commercialization of the Sibased LIB anodes. BTR New Energy Materials Inc. developed high capacity $\left(600-650 \mathrm{~mA} \mathrm{~h} \mathrm{~g}^{-1}\right) \mathrm{Si}$ based composite material with an initial CE of $89 \%-90 \%$. Nexeon designed silicon based composite powder for hybrid or low loading anode electrodes (recommended use up to $10 \mathrm{wt} . \%$ loading) with capacities up to $400-450 \mathrm{~mA} \mathrm{~h} \mathrm{~g}^{-1}$. Further industrialization should focus on the energy density of full cell in practical application instead of half coin cells, and several critical concerns should be settled.

Firstly, the silicon volume expansion will become more serious when used in practical application that usually needs a higher current density. Mass loading of the Si-based electrode is a key issue to realize the decent energy output in real battery applications. Unfortunately, the real mass loading density is not high enough. Secondly, the $\mathrm{CE}$ is also crucial for practical battery applications. Normally, the initial CE of the Si-based anode is typically in the range of $50 \%-90 \%$ (Table 1), which still cannot meet the need for practical battery applications. Thirdly, the consistency and cost of the silicon anode materials are far from meeting scale industrial application requirements. The low yield and complicated fabrication process or harsh synthesis condition lead to the high price of silicon nanomaterials. Moreover, pairing with high capacity $\mathrm{Ni}$-rich or $\mathrm{Li}$-rich cathode is preferred to realize the commercialization of silicon-based anodes. However, the internal failure mechanism is more complex than that of half cells.

\section{CONCLUSIONS AND PERSPECTIVES}

Silicon is one of the most promising anode materials for next generation lithium batteries owing to its ultrahigh capacity, proper working potential, low-cost and high abundance. However, the inherent large volume expansion, unstable SEI layer and poor conductivity of Si have hindered its further practical application. In this review, we discussed and summarized the rational material design principles, the recent advances in design and synthesis of silicon anode structures and in-situ characterization techniques that facilitate the deep understanding of lithiation process and capacity degradation mechanism of silicon anode in LIBs.

Over the past decades, the above issues have been addressed through various methods. Especially, structural design is believed to be the most effective solution and it can be summarized as follow: construction of silicon nanostructures (downsizing the $\mathrm{Si}$ to nanoscale with various morphologies, dimensions and porous structures), fabrication of silicon composite (compositing with carbon, metal, metal oxide and conductive polymer). Early studies have shown that nanosized silicon can effectively inhibit the volume expansion and pulverization phenomenon. However, the larger specific surface area results in much more formation of SEI and consumption of Li ion, which further leads to low CE. Compositing silicon with carbon, the conductivity of silicon-based materials can be effectively improved, which can further improve the rate performance. On the other hand, the introduced carbon materials can buffer the volume change effect of silicon and avoid direct contact between silicon and electrolyte, prevent uncontrolled SEI film formation at once, which significantly improve the electrochemical properties of silicon-based materials. However, the tap density and mass loading of silicon are still far from satisfaction to achieve higher energy density. Silicon/metal and conductive polymer composite almost share a similar working concept with silicon/carbon, while these materials are hindered by the high cost, limited resource and complicated preparation process for large scale application.

For now, coating or modifying Si with carbon has been highlighted and demonstrated the most common and effective way. Meantime, the $\mathrm{Si} / \mathrm{C}$ has been partially commercialized. However, real breakthroughs are still in urgent need to come. Firstly, improving the tap density and mass loading of silicon to ensure the volume and specific energy density is the most important issue to meet the commercial need. Secondly, the synthesis of nanomaterials is in high cost, which is of key importance for widespread commercial applications. Thirdly, it is hard to ensure the consistency of materials when it comes to industrial production. So, it is crucial to explore facile and environmentally friendly routes to obtain low-cost, large-scale and high-quality silicon/carbon anode.

We have witnessed the fast development of several in situ electrochemistry techniques in the recent years, such as in situ TEM, SEM, AFM, XRD and NMR, for the evaluation of real-time energy storage behaviors of silicon-based batteries. The new insights contribute to a better understanding of device failure and monitoring on the electrode morphological change, phase evolution, SEI formation and evolution and mechanical property, guiding future material optimization and innovation. However, the present in situ cells are incapable of completely mimicking real battery-operation conditions, and 
Table 1 Summary of synthesis method and electrochemical characteristics of selected Si-based anode nanostructure

\begin{tabular}{|c|c|c|c|c|c|}
\hline Active materials & Synthesis method & $\begin{array}{l}\text { Mass loading } \\
\left(\mathrm{mg} \mathrm{cm}^{-2}\right)\end{array}$ & $\begin{array}{l}\text { First cycle capacity } \\
\left(\mathrm{mA} \mathrm{h} \mathrm{g}^{-1}\right)\left(\mathrm{A} \mathrm{g}^{-1}\right)\end{array}$ & $\begin{array}{c}\text { Initial Coulombic } \\
\text { efficiency }\end{array}$ & $\begin{array}{l}\text { No. cycles with } \\
80 \% \text { capacity retention }\end{array}$ \\
\hline Mic-Si/graphene cage [81] & Ultrasonication & 0.8 & $3300(0.05)$ & $93.2 \%$ & No decrease after 100 cycles \\
\hline $\begin{array}{l}\text { Hierarchically porous } \mathrm{Si} \\
{[78]}\end{array}$ & Dispersed in ethanol and cast & 1 & $1850(0.42)$ & $87 \%$ & 60 \\
\hline Si NPs [98] & Stirring for $6 \mathrm{~h}$ at $200^{\circ} \mathrm{C}$ & $2.0-2.5$ & $2500(0.05)$ & $85 \%$ & 15 \\
\hline Si NPs [92] & Solution processes & $0.3-0.4$ & $1200(1.0)$ & $70 \%$ & 180 \\
\hline SiMP [66] & Condensation reaction & $0.5-0.7$ & $3200(0.5)$ & $80 \%$ & 80 \\
\hline $\mathrm{Si} / \mathrm{C}[99]$ & CVD & 1.8 & $2277(0.42)$ & $90 \%$ & 16 \\
\hline $\mathrm{p}-\mathrm{Si} / \mathrm{C}[77]$ & Magnesiothermic reduction & 1.1 & $2500(2.6)$ & $75 \%$ & 10 \\
\hline Si nanorods [101] & Self-templating synthesis & 1.1 & $2705(0.25)$ & $60.5 \%$ & No decrease after 500 cycles \\
\hline PG-Si [102] & $\begin{array}{l}\text { Chemical reduction or } \\
\text { decompose }\end{array}$ & 1 & $1464(0.2)$ & $58 \%$ & 70 \\
\hline $\mathrm{Si} / \mathrm{C}[103]$ & CVD & $1-5$ & $3207(0.05)$ & $79 \%$ & No decrease after 100 cycles \\
\hline Mesoporous Si [104] & Pyrolysis & 0.5 & $2660(0.5)$ & $72.8 \%$ & 175 \\
\hline $\mathrm{SiO} / \mathrm{G} / \mathrm{C}[105]$ & Heat treatment & 2 & $905(1.0)$ & $68.1 \%$ & No decrease after 200 cycles \\
\hline $\mathrm{SiO}_{2}$ nanowires [106] & Pyrolysis & 1.0 & $2215(0.5)$ & $88 \%$ & 10 \\
\hline Si@C [107] & Pyrolysis & 0.5 & $2936(0.09)$ & $85 \%$ & 10 \\
\hline $\mathrm{MHSiO}_{2} @ \mathrm{C}[108]$ & Pyrolysis & $1.73-1.91$ & $880(0.5)$ & $68 \%$ & 5 \\
\hline $\mathrm{Si} @ \mathrm{TiO}_{2}[109]$ & Sonication and pyrolysis & 0.8 & $1562(2.1)$ & $65.8 \%$ & 68 \\
\hline HSi@C [110] & Reduce $\mathrm{HSiO}_{2}$ & 0.7 & $1970(2.0)$ & 52.4 & No decrease after 200 cycles \\
\hline CNT-Si [111] & Etching by HF & 1.3 & $2213(0.42)$ & $83.4 \%$ & 50 \\
\hline Mesoporous Si [112] & Pyrolysis & $0.7-1.2$ & $2789(0.36)$ & $64.1 \%$ & 70 \\
\hline Ferrosilicon [59] & High energy ball milling & 0.5 & $1250(0.4)$ & $88 \%$ & No decrease after 100 cycles \\
\hline $\mathrm{Si} / \mathrm{C}[113]$ & Thermal decomposition & $0.6-1$ & $1992(0.2)$ & $60 \%$ & 110 \\
\hline Si-SHP [114] & Ultrasonication & 1.13 & $2850(0.1)$ & $80 \%$ & 25 \\
\hline Si@GO [115] & $3 \mathrm{D}$ tomography & 1.2 & $2900(0.42)$ & $94 \%$ & 70 \\
\hline Si/graphene [116] & Mechanical stirring & 2 & $879(0.05)$ & $58.6 \%$ & 6 \\
\hline
\end{tabular}

detailed information about the electrochemical mechanisms, ion and electron kinetic transport at the electrode/ electrolyte interface are still absent due to their complexity. On the other hand, one in situ characterization technique can obtain only single information, which may give rise to inconsistency of the observed information and lead to further disagreements on the conclusions. Thus, it is highly desirable to develop integrated technique that can get all the information at the same time so that a more complete picture can be drawn than one technique acting alone. This requires a fast data-collection rate, which is not possible using most of the present tools and expected to motivate future studies.

Despite some great achievements for enhancing battery performance, other challenges of $\mathrm{Si}$ anode such as the scalable synthesis and the compatablility to combine with novel components still remain for the commercialization of Si-based battery. Exploration of other interrelated strategies, such as electrolytes, electrolyte additives, new current collector and binders, will also help to improve the electrochemical performance of lithium-based rechargeable batteries using nanostructured silicon electrode materials. With the fast development and extensive research interest of science and technology in this area, the obstacles that hinder the practical applications of $\mathrm{Si}$ anode material will be addressed successfully in the near future.

Received 13 May 2019; accepted 22 June 2019; published online 30 July 2019

1 Chen J, Cheng F. Combination of lightweight elements and nanostructured materials for batteries. Acc Chem Res, 2009, 42: 713-723

2 Zhang K, Hu Z, Tao Z, et al. Inorganic \& organic materials for rechargeable Li batteries with multi-electron reaction. Sci China Mater, 2014, 57: 42-58

3 Schmuch R, Wagner R, Hörpel G, et al. Performance and cost of 
materials for lithium-based rechargeable automotive batteries. Nat Energy, 2018, 3: 267-278

4 Hao J, Liu H, Ji Y, et al. Synthesis and electrochemical performance of Sn-doped $\mathrm{LiNi}_{0.5} \mathrm{Mn}_{1.5} \mathrm{O}_{4}$ cathode material for highvoltage lithium-ion batteries. Sci China Mater, 2017, 60: 315-323

$5 \mathrm{Li} \mathrm{Y,} \mathrm{Li} \mathrm{X,} \mathrm{Wang} \mathrm{Z,} \mathrm{et} \mathrm{al.} \mathrm{An} \mathrm{Ostwald} \mathrm{ripening} \mathrm{route} \mathrm{towards} \mathrm{Ni-}$ rich layered cathode material with cobalt-rich surface for lithium ion battery. Sci China Mater, 2018, 61: 719-727

6 Li H, Zhou P, Liu F, et al. Stabilizing nickel-rich layered oxide cathodes by magnesium doping for rechargeable lithium-ion batteries. Chem Sci, 2019, 10: 1374-1379

7 Chen L, Jiang $\mathrm{H}, \mathrm{Hu} \mathrm{Y}$, et al. In-situ growth of ultrathin $\mathrm{MoS}_{2}$ nanosheets on sponge-like carbon nanospheres for lithium-ion batteries. Sci China Mater, 2018, 61: 1049-1056

8 Cheng F, Liang J, Tao Z, et al. Functional materials for rechargeable batteries. Adv Mater, 2011, 23: 1695-1715

9 Lu Y, Zhang Q, Chen J. Recent progress on lithium-ion batteries with high electrochemical performance. Sci China Chem, 2019, 62: $533-548$

10 Zheng S, Sun H, Yan B, et al. High-capacity organic electrode material calix[4] quinone/CMK-3 nanocomposite for lithium batteries. Sci China Mater, 2018, 61: 1285-1290

11 Lu Y, Hou X, Miao L, et al. Cyclohexanehexone with ultrahigh capacity as cathode materials for lithium-ion batteries. Angew Chem Int Ed, 2019, 58: 7020-7024

12 Zhu Y, Cao T, Li Z, et al. Two-dimensional $\mathrm{SnO}_{2} /$ graphene heterostructures for highly reversible electrochemical lithium storage. Sci China Mater, 2018, 61: 1527-1535

13 Lou L, Kong X, Zhu T, et al. Facile fabrication of interconnectedmesoporous $\mathrm{T}-\mathrm{Nb}_{2} \mathrm{O}_{5}$ nanofibers as anodes for lithium-ion batteries. Sci China Mater, 2019, 62: 465-473

14 Rahman MA, Song G, Bhatt AI, et al. Nanostructured silicon anodes for high-performance lithium-ion batteries. Adv Funct Mater, 2016, 26: 647-678

15 Long W, Fang B, Ignaszak A, et al. Biomass-derived nanostructured carbons and their composites as anode materials for lithium ion batteries. Chem Soc Rev, 2017, 46: 7176-7190

16 Sun Y, Liu N, Cui Y. Promises and challenges of nanomaterials for lithium-based rechargeable batteries. Nat Energy, 2016, 1: 16071-16082

17 Liu Z, Yu Q, Zhao Y, et al. Silicon oxides: a promising family of anode materials for lithium-ion batteries. Chem Soc Rev, 2019, 48: 285-309

18 Ma J, Sung J, Hong J, et al. Towards maximized volumetric capacity via pore-coordinated design for large-volume-change lithium-ion battery anodes. Nat Commun, 2019, 10: 475-484

19 Michan AL, Divitini G, Pell AJ, et al. Solid electrolyte interphase growth and capacity loss in silicon electrodes. J Am Chem Soc, 2016, 138: 7918-7931

20 McDowell MT, Woo Lee S, Wang C, et al. The effect of metallic coatings and crystallinity on the volume expansion of silicon during electrochemical lithiation/delithiation. Nano Energy, 2012, 1: 401-410

21 Qi W, Shapter JG, Wu Q, et al. Nanostructured anode materials for lithium-ion batteries: principle, recent progress and future perspectives. J Mater Chem A, 2017, 5: 19521-19540

22 Zuo X, Zhu J, Müller-Buschbaum P, et al. Silicon based lithiumion battery anodes: A chronicle perspective review. Nano Energy, 2017, 31: 113-143

23 Su X, Wu Q, Li J, et al. Silicon-based nanomaterials for lithium- ion batteries: A review. Adv Energy Mater, 2014, 4: 1300882

$24 \mathrm{Gu} \mathrm{M}, \mathrm{He} \mathrm{Y,} \mathrm{Zheng} \mathrm{J,} \mathrm{et} \mathrm{al.} \mathrm{Nanoscale} \mathrm{silicon} \mathrm{as} \mathrm{anode} \mathrm{for} \mathrm{Li-ion}$ batteries: The fundamentals, promises, and challenges. Nano Energy, 2015, 17: 366-383

25 https://materialsproject.org

26 Tao Z, Wang H, Chen J. Si-based materials as the anode of lithium-ion batteries. Prog Chem, 2011, 23: 318-327

27 Jeong S, Lee JP, Ko M, et al. Etched graphite with internally grown Si nanowires from pores as an anode for high density Liion batteries. Nano Lett, 2013, 13: 3403-3407

28 Axel H, Schäfer H, Weiss A. Zur Kenntnis der Phase $\mathrm{Li}_{22} \mathrm{Si}_{5}$. Z für Naturforschung B, 1966, 21: 115-117

29 Dey AN. Electrochemical alloying of lithium in organic electrolytes. J Electrochem Soc, 1971, 118: 1547-1549

30 Seefurth RN, Sharma RA. Investigation of lithium utilization from a lithium-silicon electrode. J Electrochem Soc, 1977, 124: 1207-1214

31 Wilson AM, Way BM, Dahn JR, et al. Nanodispersed silicon in pregraphitic carbons. J Appl Phys, 1995, 77: 2363-2369

32 Li H, Huang XJ, Chen LQ, et al. A high capacity nano Si composite anode material for lithium rechargeable batteries. Electrochem Solid-State Lett, 1999, 2: 547-549

33 Morales AM, Lieber CM. A laser ablation method for the synthesis of crystalline semiconductor nanowires. Science, 1998, 279: 208-211

34 Guo ZP, Wang JZ, Liu HK, et al. Study of silicon/polypyrrole composite as anode materials for Li-ion batteries. J Power Sources, 2005, 146: 448-451

35 Park MH, Kim MG, Joo J, et al. Silicon nanotube battery anodes. Nano Lett, 2009, 9: 3844-3847

36 Kim H, Han B, Choo J, et al. Three-dimensional porous silicon particles for use in high-performance lithium secondary batteries. Angew Chem Int Ed, 2008, 47: 10151-10154

37 Ma H, Cheng F, Chen JY, et al. Nest-like silicon nanospheres for high-capacity lithium storage. Adv Mater, 2007, 19: 4067-4070

38 Chan CK, Patel RN, O'Connell MJ, et al. Solution-grown silicon nanowires for lithium-ion battery anodes. ACS Nano, 2010, 4: $1443-1450$

39 Yao Y, McDowell MT, Ryu I, et al. Interconnected silicon hollow nanospheres for lithium-ion battery anodes with long cycle life. Nano Lett, 2011, 11: 2949-2954

$40 \mathrm{Wu} \mathrm{H}$, Chan G, Choi JW, et al. Stable cycling of double-walled silicon nanotube battery anodes through solid-electrolyte interphase control. Nat Nanotech, 2012, 7: 310-315

41 Tritsaris GA, Kaxiras E, Meng S, et al. Adsorption and diffusion of lithium on layered silicon for Li-ion storage. Nano Lett, 2013, 13: $2258-2263$

42 Liu N, Lu Z, Zhao J, et al. A pomegranate-inspired nanoscale design for large-volume-change lithium battery anodes. Nat Nanotech, 2014, 9: 187-192

43 Baggetto L, Danilov D, Notten PHL. Honeycomb-structured silicon: remarkable morphological changes induced by electrochemical (de)lithiation. Adv Mater, 2011, 23: 1563-1566

44 Lin N, Han Y, Wang L, et al. Preparation of nanocrystalline silicon from $\mathrm{SiCl}_{4}$ at $200^{\circ} \mathrm{C}$ in molten salt for high-performance anodes for lithium ion batteries. Angew Chem Int Ed, 2015, 54: 3822-3825

45 Jia H, Gao P, Yang J, et al. Novel three-dimensional mesoporous silicon for high power lithium-ion battery anode material. Adv Energy Mater, 2011, 1: 1036-1039 
46 Yoo JK, Kim J, Jung YS, et al. Scalable fabrication of silicon nanotubes and their application to energy storage. Adv Mater, 2012, 24: 5452-5456

47 Li X, Gu M, Hu S, et al. Mesoporous silicon sponge as an antipulverization structure for high-performance lithium-ion battery anodes. Nat Commun, 2014, 5: 4105-4111

48 Peng B, Cheng FY, Tao ZL, et al. Lithium transport at silicon thin film: Barrier for high-rate capability anode. J Chem Phys, 2010, 133: 034701

49 Ryu J, Chen T, Bok T, et al. Mechanical mismatch-driven rippling in carbon-coated silicon sheets for stress-resilient battery anodes. Nat Commun, 2018, 9: 2924-2931

50 Shang H, Zuo Z, Yu L, et al. Low-temperature growth of allcarbon graphdiyne on a silicon anode for high-performance lithium-ion batteries. Adv Mater, 2018, 30: 1801459

51 Han Y, Zou J, Li Z, et al. Si@void@C nanofibers fabricated using a self-powered electrospinning system for lithium-ion batteries. ACS Nano, 2018, 12: 4835-4843

$52 \mathrm{Xu}$ Q, Li JY, Sun JK, et al. Watermelon-inspired Si/C microspheres with hierarchical buffer structures for densely compacted lithium-ion battery anodes. Adv Energy Mater, 2017, 7: 1601481

53 Xu R, Wang G, Zhou T, et al. Rational design of Si@carbon with robust hierarchically porous custard-apple-like structure to boost lithium storage. Nano Energy, 2017, 39: 253-261

54 Son IH, Park JH, Park S, et al. Graphene balls for lithium rechargeable batteries with fast charging and high volumetric energy densities. Nat Commun, 2017, 8: 1561-1571

55 Ko M, Chae S, Ma J, et al. Scalable synthesis of silicon-nanolayerembedded graphite for high-energy lithium-ion batteries. Nat Energy, 2016, 1: 16113-16120

56 Yin $\mathrm{S}$, Zhao D, Ji Q, et al. Si/Ag/C nanohybrids with in situ incorporation of super-small silver nanoparticles: tiny amount, huge impact. ACS Nano, 2018, 12: 861-875

57 Kim GT, Kennedy T, Brandon M, et al. Behavior of germanium and silicon nanowire anodes with ionic liquid electrolytes. ACS Nano, 2017, 11: 5933-5943

58 Jin $\mathrm{Y}$, Tan $\mathrm{Y}, \mathrm{Hu} \mathrm{X}$, et al. Scalable production of the silicon-tin yin-yang hybrid structure with graphene coating for high performance lithium-ion battery anodes. ACS Appl Mater Interfaces, 2017, 9: 15388-15393

59 Li H, Cheng F, Zhu Z, et al. Preparation and electrochemical performance of copper foam-supported amorphous silicon thin films for rechargeable lithium-ion batteries. J Alloys Compd, 2011, 509: 2919-2923

$60 \mathrm{Ma} \mathrm{T}, \mathrm{Yu} \mathrm{X}, \mathrm{Li} \mathrm{H}$, et al. High volumetric capacity of hollow structured $\mathrm{SnO}_{2} @ \mathrm{Si}$ nanospheres for lithium-ion batteries. Nano Lett, 2017, 17: 3959-3964

61 Li Z, Wang F, Wang X. Hierarchical branched vanadium oxide nanorod@Si nanowire architecture for high performance supercapacitors. Small, 2017, 13: 1603076

62 Park E, Yoo H, Lee J, et al. Dual-size silicon nanocrystalembedded $\mathrm{SiO}_{x}$ nanocomposite as a high-capacity lithium storage material. ACS Nano, 2015, 9: 7690-7696

63 Luo L, Yang H, Yan P, et al. Surface-coating regulated lithiation kinetics and degradation in silicon nanowires for lithium ion battery. ACS Nano, 2015, 9: 5559-5566

64 Liu J, Zhang Q, Zhang T, et al. A robust ion-conductive biopolymer as a binder for $\mathrm{Si}$ anodes of lithium-ion batteries. Adv Funct Mater, 2015, 25: 3599-3605

65 Chen Y, Zeng S, Qian J, et al. $\mathrm{Li}^{+}$-conductive polymer-embedded
nano-Si particles as anode material for advanced Li-ion batteries. ACS Appl Mater Interfaces, 2014, 6: 3508-3512

66 Wang $\mathrm{C}, \mathrm{Wu} \mathrm{H}$, Chen $\mathrm{Z}$, et al. Self-healing chemistry enables the stable operation of silicon microparticle anodes for high-energy lithium-ion batteries. Nat Chem, 2013, 5: 1042-1048

67 Szczech JR, Jin S. Nanostructured silicon for high capacity lithium battery anodes. Energy Environ Sci, 2011, 4: 56-72

68 Kim H, Seo M, Park MH, et al. A critical size of silicon nanoanodes for lithium rechargeable batteries. Angew Chem Int Ed, 2010, 49: 2146-2149

69 Liu XH, Zhong L, Huang S, et al. Size-dependent fracture of silicon nanoparticles during lithiation. ACS Nano, 2012, 6: 15221531

70 Lin N, Han Y, Zhou J, et al. A low temperature molten salt process for aluminothermic reduction of silicon oxides to crystalline Si for Li-ion batteries. Energy Environ Sci, 2015, 8: 31873191

71 Chan CK, Peng H, Liu G, et al. High-performance lithium battery anodes using silicon nanowires. Nat Nanotech, 2008, 3: 31-35

72 Wang X, Huang L, Zhang Y, et al. Novel silicon nanowire film on copper foil as high performance anode for lithium-ion batteries. Ionics, 2018, 24: 373-378

73 Chen S, Chen Z, Xu X, et al. Scalable 2D mesoporous silicon nanosheets for high-performance lithium-ion battery anode. Small, 2018, 14: 1703361

74 Zhang X, Qiu X, Kong D, et al. Silicene flowers: A dual stabilized silicon building block for high-performance lithium battery anodes. ACS Nano, 2017, 11: 7476-7484

75 Liu J, Yang Y, Lyu P, et al. Few-layer silicene nanosheets with superior lithium-storage properties. Adv Mater, 2018, 30: 1800838

76 Zuo X, Xia Y, Ji Q, et al. Self-templating construction of 3D hierarchical macro-/mesoporous silicon from $0 \mathrm{D}$ silica nanoparticles. ACS Nano, 2017, 11: 889-899

77 Jia $\mathrm{H}$, Zheng J, Song J, et al. A novel approach to synthesize micrometer-sized porous silicon as a high performance anode for lithium-ion batteries. Nano Energy, 2018, 50: 589-597

78 Xiao Q, Gu M, Yang H, et al. Inward lithium-ion breathing of hierarchically porous silicon anodes. Nat Commun, 2015, 6: 8844-8851

79 Magasinski A, Dixon P, Hertzberg B, et al. High-performance lithium-ion anodes using a hierarchical bottom-up approach. Nat Mater, 2010, 9: 353-358

80 Nie P, Liu X, Fu R, et al. Mesoporous silicon anodes by using polybenzimidazole derived pyrrolic $\mathrm{N}$-enriched carbon toward high-energy Li-ion batteries. ACS Energy Lett, 2017, 2: 12791287

81 Li Y, Yan K, Lee HW, et al. Growth of conformal graphene cages on micrometre-sized silicon particles as stable battery anodes. Nat Energy, 2016, 1: 15029-15036

82 Guo S, Hu X, Hou Y, et al. Tunable synthesis of yolk-shell porous silicon@carbon for optimizing Si/C-based anode of lithium-ion batteries. ACS Appl Mater Interfaces, 2017, 9: 42084-42092

83 Chen S, Shen L, van Aken PA, et al. Dual-functionalized double carbon shells coated silicon nanoparticles for high performance lithium-ion batteries. Adv Mater, 2017, 29: 1605650

84 An W, Gao B, Mei S, et al. Scalable synthesis of ant-nest-like bulk porous silicon for high-performance lithium-ion battery anodes. Nat Commun, 2019, 10: 1447-1457

85 Li B, Yang S, Li S, et al. From commercial sponge toward 3D 
graphene-silicon networks for superior lithium storage. Adv Energy Mater, 2015, 5: 1500289

86 Song H, Wang HX, Lin Z, et al. Highly connected silicon-copper alloy mixture nanotubes as high-rate and durable anode materials for lithium-ion batteries. Adv Funct Mater, 2016, 26: 524-531

87 Yang Y, Liu S, Bian X, et al. Morphology- and porosity-tunable synthesis of 3D nanoporous SiGe alloy as a high-performance lithium-ion battery anode. ACS Nano, 2018, 12: 2900-2908

88 Zhang Q, Chen H, Luo L, et al. Harnessing the concurrent reaction dynamics in active $\mathrm{Si}$ and $\mathrm{Ge}$ to achieve high performance lithium-ion batteries. Energy Environ Sci, 2018, 11: 669-681

89 Fang S, Shen L, Xu G, et al. Rational design of void-involved $\mathrm{Si} @ \mathrm{TiO}_{2}$ nanospheres as high-performance anode material for lithium-ion batteries. ACS Appl Mater Interfaces, 2014, 6: 64976503

90 Yang J, Wang Y, Li W, et al. Amorphous $\mathrm{TiO}_{2}$ shells: a vital elastic buffering layer on silicon nanoparticles for high-performance and safe lithium storage. Adv Mater, 2017, 29: 1700523

91 Wang $\mathrm{C}$, Han $\mathrm{Y}, \mathrm{Li} \mathrm{S}$, et al. Thermal lithiated- $\mathrm{TiO}_{2}$ : A robust and electron-conducting protection layer for $\mathrm{Li}-\mathrm{Si}$ alloy anode. ACS Appl Mater Interfaces, 2018, 10: 12750-12758

92 Wu H, Yu G, Pan L, et al. Stable Li-ion battery anodes by in-situ polymerization of conducting hydrogel to conformally coat silicon nanoparticles. Nat Commun, 2013, 4: 1943-1948

$93 \mathrm{Du} \mathrm{FH}, \mathrm{Li} \mathrm{B}, \mathrm{Fu} \mathrm{W}$, et al. Surface binding of polypyrrole on porous silicon hollow nanospheres for Li-ion battery anodes with high structure stability. Adv Mater, 2014, 26: 6145-6150

94 Kovalenko I, Zdyrko B, Magasinski A, et al. A major constituent of brown algae for use in high-capacity Li-ion batteries. Science, 2011, 334: 75-79

95 Choi S, Kwon TW, Coskun A, et al. Highly elastic binders integrating polyrotaxanes for silicon microparticle anodes in lithium ion batteries. Science, 2017, 357: 279-283

96 Munaoka T, Yan X, Lopez J, et al. Ionically conductive selfhealing binder for low cost $\mathrm{Si}$ microparticles anodes in Li-ion batteries. Adv Energy Mater, 2018, 8: 1703138

97 Zeng W, Wang L, Peng X, et al. Enhanced ion conductivity in conducting polymer binder for high-performance silicon anodes in advanced lithium-ion batteries. Adv Energy Mater, 2018, 8: 1702314-1702321

98 Liu Y, Tai Z, Zhou T, et al. An all-integrated anode via interlinked chemical bonding between double-shelled-yolk-structured silicon and binder for lithium-ion batteries. Adv Mater, 2017, 29: 1703028-1703038

99 Zhao J, Lu Z, Liu N, et al. Dry-air-stable lithium silicide-lithium oxide core-shell nanoparticles as high-capacity prelithiation reagents. Nat Commun, 2014, 5: 5088-5095

100 He Y, Xiang K, Zhou W, et al. Folded-hand silicon/carbon threedimensional networks as a binder-free advanced anode for highperformance lithium-ion batteries. Chem Eng J, 2018, 353: 666678

101 Chen Q, Zhu R, Liu S, et al. Self-templating synthesis of silicon nanorods from natural sepiolite for high-performance lithiumion battery anodes. J Mater Chem A, 2018, 6: 6356-6362

102 Wei L, Hou Z, Wei H. Porous sandwiched graphene/silicon anodes for lithium storage. Electrochim Acta, 2017, 229: 445-451

103 Wang W, Favors Z, Li C, et al. Silicon and carbon nanocomposite spheres with enhanced electrochemical performance for full cell lithium ion batteries. Sci Rep, 2017, 7: 44838-44846

104 Sun L, Wang F, Su T, et al. Room-temperature solution synthesis of mesoporous silicon for lithium ion battery anodes. ACS Appl Mater Interfaces, 2017, 9: 40386-40393

105 Pan Q, Zuo P, Mu T, et al. Improved electrochemical performance of micro-sized $\mathrm{SiO}$-based composite anode by prelithiation of stabilized lithium metal powder. J Power Sources, 2017, 347: 170-177

106 Li Z, He Q, He L, et al. Self-sacrificed synthesis of carbon-coated $\mathrm{SiO}_{x}$ nanowires for high capacity lithium ion battery anodes. J Mater Chem A, 2017, 5: 4183-4189

107 Li C, Liu C, Wang W, et al. Silicon derived from glass bottles as anode materials for lithium ion full cell batteries. Sci Rep, 2017, 7: 917-927

108 An W, Fu J, Su J, et al. Mesoporous hollow nanospheres consisting of carbon coated silica nanoparticles for robust lithiumion battery anodes. J Power Sources, 2017, 345: 227-236

109 Jin Y, Li S, Kushima A, et al. Self-healing SEI enables full-cell cycling of a silicon-majority anode with a coulombic efficiency exceeding 99.9\%. Energy Environ Sci, 2017, 10: 580-592

110 Fang S, Tong Z, Nie P, et al. Raspberry-like nanostructured silicon composite anode for high-performance lithium-ion batteries. ACS Appl Mater Interfaces, 2017, 9: 18766-18773

111 Choi MJ, Xiao Y, Hwang JY, et al. Novel strategy to improve the Li-storage performance of micro silicon anodes. J Power Sources, 2017, 348: 302-310

112 Liang J, Li X, Hou Z, et al. A deep reduction and partial oxidation strategy for fabrication of mesoporous $\mathrm{Si}$ anode for lithium ion batteries. ACS Nano, 2016, 10: 2295-2304

113 Kim JS, Pfleging W, Kohler R, et al. Three-dimensional silicon/ carbon core-shell electrode as an anode material for lithium-ion batteries. J Power Sources, 2015, 279: 13-20

114 Chen Z, Wang C, Lopez J, et al. High-areal-capacity silicon electrodes with low-cost silicon particles based on spatial control of self-healing binder. Adv Energy Mater, 2015, 5: 1401826

115 Ge M, Lu Y, Ercius P, et al. Large-scale fabrication, 3D tomography, and lithium-ion battery application of porous silicon. Nano Lett, 2014, 14: 261-268

116 Xue L, Fu K, Li Y, et al. Si/C composite nanofibers with stable electric conductive network for use as durable lithium-ion battery anode. Nano Energy, 2013, 2: 361-367

117 Becker CR, Strawhecker KE, McAllister QP, et al. In situ atomic force microscopy of lithiation and delithiation of silicon nanostructures for lithium ion batteries. ACS Nano, 2013, 7: 9173-9182

118 Liu XR, Deng X, Liu RR, et al. Single nanowire electrode electrochemistry of silicon anode by in situ atomic force microscopy: solid electrolyte interphase growth and mechanical properties. ACS Appl Mater Interfaces, 2014, 6: 20317-20323

119 Kumar R, Tokranov A, Sheldon BW, et al. In situ and operando investigations of failure mechanisms of the solid electrolyte interphase on silicon electrodes. ACS Energy Lett, 2016, 1: 689-697

120 McDowell MT, Lee SW, Harris JT, et al. In situ TEM of two-phase lithiation of amorphous silicon nanospheres. Nano Lett, 2013, 13 : 758-764

121 Lee SW, Lee HW, Ryu I, et al. Kinetics and fracture resistance of lithiated silicon nanostructure pairs controlled by their mechanical interaction. Nat Commun, 2015, 6: 7533-7539

122 Wang CM, Li X, Wang Z, et al. In situ TEM investigation of congruent phase transition and structural evolution of nanostructured silicon/carbon anode for lithium ion batteries. Nano Lett, 2012, 12: 1624-1632

123 He Y, Piper DM, Gu M, et al. In situ transmission electron mi- 
croscopy probing of native oxide and artificial layers on silicon nanoparticles for lithium ion batteries. ACS Nano, 2014, 8: 11816-11823

124 Yu WJ, Liu C, Hou PX, et al. Lithiation of silicon nanoparticles confined in carbon nanotubes. ACS Nano, 2015, 9: 5063-5071

125 Wang X, Fan F, Wang J, et al. High damage tolerance of electrochemically lithiated silicon. Nat Commun, 2015, 6: 8417-8423

126 Ogata K, Salager E, Kerr CJ, et al. Revealing lithium-silicide phase transformations in nano-structured silicon-based lithium ion batteries via in situ NMR spectroscopy. Nat Commun, 2014, 5: 3217

127 Misra S, Liu N, Nelson J, et al. In situ X-ray diffraction studies of (de)lithiation mechanism in silicon nanowire anodes. ACS Nano, 2012, 6: 5465-5473

128 Tardif S, Pavlenko E, Quazuguel L, et al. Operando Raman spectroscopy and synchrotron X-ray diffraction of lithiation/delithiation in silicon nanoparticle anodes. ACS Nano, 2017, 11: 11306-11316

Acknowledgements This work was supported by the National Programs for Nano-Key Project (2017YFA0206700), the National Key R\&D Program of China (2018YFB1502100), the National Natural Science Foundation of China (21835004), 111 Project from the Ministry of Education of China (B12015) and the Fundamental Research Funds for the Central Universities, Nankai University (63191711 and 63191416).

Author contributions Yan Z, Cheng F and Chen J proposed the topic and outline of the manuscript. Chen $\mathrm{X}, \mathrm{Li} \mathrm{H}$, and Yan $\mathrm{Z}$ collected the related information and wrote the manuscript. All authors contributed to the general discussion and revision.

Conflict of interest The authors declare that they have no conflict of interest.

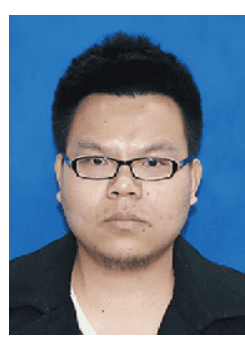

Xiang Chen is a $\mathrm{PhD}$ candidate at the College of Chemistry, Nankai University. He received his Bachelor degree (2011) and Master degree (2014) from Haerbin Normal University. He moved to Nankai University in 2016. His research focuses on lithium and zinc based batteries.

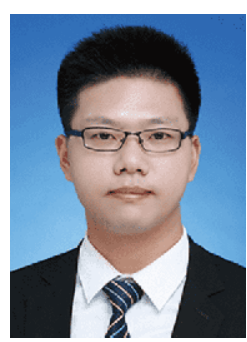

Zhenhua Yan is a lecturer at the College of Chemistry, Nankai University. He received his Bachelor degree (2011) and Master degree (2014) from Liaocheng University. He obtained his $\mathrm{PhD}$ degree from Nankai University in 2018, under the supervision of Prof. Fangyi Cheng and Prof. Jun Chen. His current research interest is nanomaterials for batteries and electrocatalysis.

\section{锂离子电池中硅负极材料的设计及机理分析研究 进展}

陈祥, 李海霞, 严振华 ${ }^{*}$, 程方益, 陈军

摘要 硅基负极具有理论容量高、工作电位低、环境友好、丰度 高等优点, 被认为是下一代锂离子电池中最有希望替代石墨负极 的材料之一. 然而, 硅负极在脱嵌锂过程中体积膨胀大、界面反应 剧烈是制约其进一步实际应用的关键问题. 合理设计硅纳米结构 有助于解决这些问题. 本文首先介绍了高容量锂电池用纳米硅负 极的基本科学问题, 然后重点介绍了其设计、制备、原位表征和 失效机理等方面的最新进展, 总结到目前为止关键的经验以促进 硅负极在实际锂电池中的应用. 最后展望了硅负极未来的发展和 挑战. 